\title{
PALOMAR 13: AN UNUSUAL STELLAR SYSTEM IN THE GALACTIC HALO ${ }^{1}$

\author{
PATRICK CÔTÉ \\ Department of Physics and Astronomy, Rutgers University, New Brunswick, NJ 08854; pcote@physics.rutgers.edu
}

\author{
S. G. DJORGOvski \\ California Institute of Technology, 1200 East California Boulevard, MS 105-24, Pasadena, CA 91125; \\ george@astro.caltech.edu \\ G. MEYLAN ${ }^{2}$ \\ European Southern Observatory, Karl-Schwarzschild-Strasse 2, 85748 Garching, Germany; \\ and Space Telescope Science Institute, 3700 San Martin Drive, Baltimore, MD 21218; gmeylan@stsci.edu
}

SANDRA CASTRO

California Institute of Technology, 1200 East California Boulevard, MS 105-24, Pasadena, CA 91125; smc@astro.caltech.edu AND

J. K. MCCARTHY

California Institute of Technology, 1200 East California Boulevard, MS 105-24, Pasadena, CA 91125; and PixelVision, 4952 Warner Avenue, Suite 300, Huntington Beach, CA 92649; jkmccarthy@pacbell.net Received 2001 November 9; accepted 2002 April 1

\begin{abstract}
We report the first results of a program to study the internal kinematics of globular clusters in the outer halo of the Milky Way. Using the Keck telescope and High Resolution Echelle Spectrometer, we have measured precise radial velocities for 30 candidate red giants in the direction of Palomar 13, an object traditionally cataloged as a compact, low-luminosity globular cluster. We have combined these radial velocities with published proper motion membership probabilities and new CCD photometry from the Keck and CanadaFrance-Hawaii telescopes to isolate a sample of 21 probable members. We find a systemic velocity of $\left\langle v_{r}\right\rangle_{s}=24.1 \pm 0.5 \mathrm{~km} \mathrm{~s}^{-1}$ and a projected, intrinsic velocity dispersion of $\sigma_{p}=2.2 \pm 0.4 \mathrm{~km} \mathrm{~s}^{-1}$. Although modest, this dispersion is nevertheless several times larger than that expected for a globular cluster of this luminosity and central concentration. Taken at face value, it implies a mass-to-light ratio of $\Upsilon_{V}=40_{-17}^{+24}$ based on the best-fit King-Michie model. The surface density profile of Palomar 13 also appears unusual compared to most Galactic globular clusters; depending upon the details of background subtraction and model-fitting, Palomar 13 either contains a substantial population of "extratidal" stars, or is considerably more spatially extended than previously suspected. The full surface density profile is equally well fitted by a King-Michie model having a high concentration and large tidal radius, or by a Navarro-Frenk-White model. We examine - and tentatively reject - a number of possible origins for the observed characteristics of Palomar 13 (e.g., velocity "jitter" among the red giant branch stars, spectroscopic binary stars, nonstandard mass functions, modified Newtonian dynamics) and conclude that the two leading explanations are either catastrophic heating during a recent perigalacticon passage or the presence of a dark matter halo. The available evidence therefore suggests that Palomar 13 is either a globular cluster that is now in the process of dissolving into the Galactic halo or a faint, dark matter-dominated stellar system.
\end{abstract}

Subject headings: Galaxy: halo — Galaxy: kinematics and dynamics — galaxies: dwarf — globular clusters: individual (Palomar 13)

\section{INTRODUCTION}

Much of our knowledge of the Galactic halo is based directly on observations of its globular clusters, the most readily identifiable halo objects. Those clusters that are located in the outer halo of the Milky Way are especially important probes of the formation and evolution of the Galaxy, as their ages and metallicities provide direct constraints

\footnotetext{
${ }^{1}$ Based on data obtained at the W. M. Keck Observatory, which is operated as a scientific partnership among the California Institute of Technology, the University of California, and NASA, and was made possible by the generous financial support of the W. M. Keck Foundation.

2 Visiting Astronomer, Canada-France-Hawaii Telescope, operated by the National Research Council of Canada, the Centre National de la Recherche Scientifique of France, and the University of Hawaii.
}

on the duration of halo formation process and on the timescale for Galactic chemical enrichment, while the shape and extent of Galaxy's dark halo is constrained by their orbital properties.

These distant clusters share their location in the outer halo with a system of nine dwarf spheroidal (dSph) galaxies. These dwarfs have Galactocentric distances in the range $24 \leq R_{G} \leq 250 \mathrm{kpc}$, an interval that includes 16 globular clusters (Mateo 1998; Harris 1996). To some extent, the dSph galaxies resemble the globular clusters in that they contain populations of old metal-poor stars. There are, however, a number of important distinctions between the two classes of objects. The central stellar densities of the dSph galaxies are much lower than those of typical globular clusters, and many of the dwarfs contain intermediate-age populations (e.g., Mateo 1998). Moreover, measurements 
of the central velocity dispersions of the $\mathrm{dSph}$ galaxies suggest that they are embedded in extended dark halos (Aaronson 1983) that are presumably nonbaryonic in nature (Feltzing, Gilmore, \& Wyse 1999), although alternative explanations have been proposed (e.g., Milgrom 1995; Klessen \& Kroupa 1998).

By contrast, color-magnitude diagrams (CMDs) of distant halo globular clusters show no sign of distinct intermediate-age populations (although there is evidence that some of the clusters may be younger than their counterparts in the inner Galaxy; e.g., Stetson et al. 1999). And while there is a preponderance of faint, low-density globular clusters in the outer halo (see, e.g., van den Bergh 1994, Fig. 1), their central stellar densities remain comfortably above those of even the most centrally concentrated $\mathrm{dSph}$ galaxies.

Dynamical studies of nearby globular clusters have further established that, unlike the $\mathrm{dSph}$ galaxies, they appear to contain little or no dark matter apart from normal stellar remnants such as white dwarfs and neutron stars (e.g., Pryor \& Meylan 1993). To date, however, a direct determination of the internal mass distribution in distant halo clusters has proven impossible since the faintness of even their most evolved red giants - coupled with their sparsely populated red giant branches (RGBs) and low surface brightnessesmake the requisite spectroscopic observations extremely challenging. For example, 12 of the 16 globular clusters with $R_{G} \gtrsim 24 \mathrm{kpc}$ have central surface brightnesses below $\mu_{V} \simeq 19.5 \mathrm{mag} \mathrm{arcsec}^{-2}$; for an assumed mass-to-light ratio of $\Upsilon_{V}=2$, these clusters are expected to have central velocity dispersions of $\sigma_{p, 0} \lesssim 2 \mathrm{~km} \mathrm{~s}^{-1}$. Thus, to study their internal dynamics, a velocity precision of $\epsilon\left(v_{r}\right) \lesssim 1 \mathrm{~km} \mathrm{~s}^{-1}$ for metal-poor stars in the range $17 \lesssim V \lesssim 20$ is required.

It is hardly surprising that these objects have been neglected in previous radial velocity surveys of Galactic globular clusters. The lone exception is NGC 2419, for which Olzewski, Pryor, \& Schommer (1993) measured velocities for 12 red giants, finding $\Upsilon_{V} \simeq 0$.7. This mass-tolight ratio is among the lowest measured for any globular cluster and argues against the notion that the cluster is embedded in a massive dark halo. On the other hand, this object is, with an absolute magnitude of $M_{V}=-9.6$ (Harris 1996), among the most luminous Galactic globular clusters and atypical of the clusters that populate the outer halo. Clearly, a dynamical study of a more representative sample of halo clusters is in order.

There are several reasons to suspect that a search for dark halos in outer halo clusters might prove profitable. First, the characteristic mass of globular clusters is similar to the Jeans mass at recombination and thus has a special cosmological significance; indeed, it has often been proposed that globular clusters may have originally been embedded in dark halos (e.g., Peebles 1984; Rosenblatt, Faber, \& Blumenthal 1988; West 1993; Cen 2001). While there is no evidence to support this conjecture, it has in practice proven surprisingly difficult to rule out (Heggie \& Hut 1996; cf. Moore 1996). For example, as clusters evolve in the Galactic tidal field, they are expected to lose mass through tidal stripping - a process that confounds efforts to reconstruct the initial mass distribution in these clusters using present-day observations. Second, there are fairly compelling reasons to believe that some Galactic dSph galaxies are dark matter-

${ }^{3}$ All mass-to-light ratios quoted in this paper are in solar units. dominated, and since globular clusters are simply the next step down in luminosity, it is therefore reasonable to expect that the same scenario that is usually invoked to explain the internal dynamics of the $\mathrm{dSph}$ galaxies (i.e., gas ejection from shallow potential wells by supernova-driven winds, followed by adiabatic expansion; Dekel \& Silk 1986) might produce end-products that resemble globular clusters if the gas ejection mechanism was particularly efficient. This possibility is especially topical in light of the emerging evidence from numerical simulations that the halos of large galaxies have been assembled hierarchically (e.g., Klypin et al. 1999; Moore et al. 1999, 2001; Côté et al. 2000), although the predicted number of low-mass dark halos greatly exceeds the observed number of dwarf galaxies in such simulations. Various means of resolving this important discrepancy have been proposed: e.g., suppressing the small-scale power in the family of cold dark matter models (see, e.g., Davé et al. 2001), or postulating the existence of low-mass " dark satellites" (see, e.g., Hirashita, Takeuchi, \& Tamura 1998; Bullock, Kravtsov, \& Weinberg 2000). There are also some reasons to believe that isolated, low-luminosity clusters may contain significant amounts of baryonic dark matter. $\mathrm{N}$-body simulations of evolving globular clusters suggest that, as clusters lose mass through dynamical evolution, the fraction of their mass contained in white dwarfs should increase steadily (Vesperini \& Heggie 1997). Since the outer halo contains a disproportionately high fraction of lowluminosity clusters, a search for dynamical evidence of extreme white dwarf populations in these clusters might prove worthwhile.

In 1998, we began a program to study the internal dynamics of distant halo globular clusters using the High Resolution Echelle Spectrometer (HIRES) at the W. M. Keck Observatory. This program, which was designed to yield the first direct measurements of the velocity dispersions and mass-to-light ratios for these clusters, was also motivated by the need for improved measurements of the systemic velocities for several of these objects (i.e., the radial velocities of four of the 16 clusters having Galactocentric distances greater than $24 \mathrm{kpc}$ are either unknown or have uncertainties of more than $20 \mathrm{~km} \mathrm{~s}^{-1}$ ). It has been noted on numerous occasions that a number of the Galactic dwarf galaxies and globular clusters fall along great circles (e.g., Lynden-Bell 1976a, 1976b; Kunkel 1979; Majewski 1994). Such streams would have obvious implications for the formation of the Galactic halo, but unambiguous evidence of their existence requires the determination of orbital parameters for the putative members. Programs are now underway to measure the proper motions of the distant halo globular clusters and dwarf satellites (e.g., Dinescu et al. 2001), and accurate radial velocities are a prerequisite for the measurement of their orbits.

In this paper, we present the first results from this program. Our sample consists of seven, predominantly lowconcentration clusters having Galactocentric distances in the range $25 \mathrm{kpc} \lesssim R_{G} \lesssim 112 \mathrm{kpc}$. An analysis of the full sample will be presented in a separate paper; here we present our findings for a single sparse object, Palomar 13. With an absolute magnitude of $M_{V} \simeq-3.8 \mathrm{mag}$, Palomar 13 is exceeded in luminosity by all but a handful of the $\sim 150$ globular clusters belonging to the Milky Way, and is one of the objects which has been identified on previous occasions as a possible member of a great stream (e.g., Lynden-Bell 1976a; Lynden-Bell \& Lynden-Bell 1995). On the basis of 
our new radial velocities and photometry, we find both the structural properties and the mass-to-light ratio of this object to be atypical of Galactic globular clusters.

\section{OBSERVATIONS AND REDUCTIONS}

\subsection{Keck and CFHT Photometry}

On the night of 1999 September 10 we used the Low Resolution Imaging Spectrometer (LRIS; Oke et al. 1995) on the Keck II telescope to obtain a series of $B V I$ images of Palomar 13. An observing log for these, and other, observations of Palomar 13 may be found in Table 1. In imaging mode, LRIS has a spatial scale of 0 ". 215 pixel $^{-1}$ and a field of view of $5 ! 8 \times 7 ! 3$. For each filter, we obtained a pair of images centered on the cluster. Exposure times were 10 and $300 \mathrm{~s}$ in $V, 20$ and $480 \mathrm{~s}$ in $B$, and 6 and $180 \mathrm{~s}$ in $I$. Conditions during the night were photometric, and the FWHM of isolated stars within the $B V I$ frames were measured to be in the range $0.7-0$ ". 8 . Images were bias-subtracted and then flatfielded in the $\operatorname{IRAF}^{4}$ environment using sky flats obtained during twilight. Instrumental magnitudes for unresolved objects in the field were derived using the DAOPHOT II software package (Stetson 1993), and calibrated with observations of several Landolt (1992) standard fields taken throughout the night. The calibration equations took the form

$$
\begin{aligned}
V & =v+a_{1}-b_{1} X_{V}+c_{1}(b-v) \\
B-V & =a_{2}-b_{2} X_{B}+c_{2}(b-v) \\
V-I & =a_{3}-b_{3} X_{I}+c_{3}(v-i) .
\end{aligned}
$$

Aperture corrections were determined separately for each filter, and a master object list was created using the photometry files for the long and short exposures. The final photometric database contains 840 objects detected with a minimum point-source signal-to-noise ratio $(\mathrm{S} / \mathrm{N})$ of $\mathrm{S} / \mathrm{N}=5$ in all three filters. A finding chart for Palomar 13 constructed from our $\mathrm{V}$ images is shown in Figure 1. All stars having measured radial velocities (see $\S 2.2)$ are identified on this image.

Images of Palomar 13 were also obtained on the night of 1999 July 14 using the $3.6 \mathrm{~m}$ Canada-France-Hawaii telescope (CFHT) and the CFH12K mosaic camera (Cuillandre et al. 2000). A series of three $600 \mathrm{~s}$ exposures in each of the $V$ and $R$ filters were taken with the cluster centered on chip 8 of the CCD mosaic. Each image was bias-subtracted and

\footnotetext{
${ }^{4}$ IRAF is distributed by the National Optical Astronomy Observatories, which are operated by the Association of Universities for Research in Astronomy, Inc., under contract to the National Science Foundation.
}

flat-fielded, and then shifted and stacked to produce a final image for each of the two filters. Isolated stars in the stacked images were found to have FWHMs of 0 ".75 in each filter. Photometry was then performed using DAOPHOT II, and the photometry lists were matched. Unfortunately, conditions were nonphotometric and it was not possible to calibrate the photometry directly. Instead, instrumental $(v)$ magnitudes for stars on chip 8 were calibrated via secondary standards in this field selected from our Keck/LRIS photometry. Thus, the photometric database from our CFHT data consists of calibrated $V$ magnitudes and instrumental $r$ magnitudes for 855 objects in this CCD field, which measures $7 ! 0 \times 14 ! 0$. This single field covers an area 2.3 times larger than that available from LRIS, although the photometric catalog is limited to the range $18.5 \mathrm{mag} \lesssim V \lesssim 23.5 \mathrm{mag}$ (as opposed to our LRIS pointsource catalog, which spans the range $15.5 \lesssim V \lesssim 24.5 \mathrm{mag}$ ).

By virtue of its remote location in the outer halo, Palomar 13 should, in principal, have played an important role in establishing the chronology of halo formation. Somewhat surprisingly, it has been the focus of only little attention in this regard. CMD studies are limited to photographic study of Ortolani, Rosino, \& Sandage (1985), the CCD study of Borissova, Markov, \& Spassova (1997), and the recent photographic/CCD study of Siegel et al. (2001). In Figure 2, we compare our LRIS magnitudes and colors for stars in common with each of these three studies. We find good agreement with the $B V$ photometry of Siegel et al. (2001), but the comparison reveals some interesting discrepancies with the earlier studies. There is evidence for a nonlinearity in the $V$ magnitudes of stars fainter than $V \sim 21$ in the Ortolani et al. (1985) study, while the Borissova et al. (1997) $V$ magnitudes show a rather large scatter with a mean offset of $\Delta V=0.22 \pm 0.06 \mathrm{mag}$. Due to the small number of red giants in this cluster-and the limited depth of previous photometric studies - the stars in common between these studies and ours span a modest range in color. As before, there is good agreement with the colors of Siegel et al. (2001). For both the Ortolani et al. (1985) and Borissova et al. (1997) data sets, we find mean offsets of $\Delta(B-V) \sim 0.1$ mag.

\subsection{HIRES Spectroscopy}

In a series of observing runs during 1998 and 1999, we used HIRES (Vogt et al. 1994) to acquire spectra for candidate red giants in the direction of seven distant halo globular clusters: Palomar 3, Palomar 4, Palomar 5, Palomar 13, Palomar 14, NGC 7492, and NGC 2419. Table 1 presents an observing log for our HIRES observations of Palomar 13, which were obtained during the course of four dark runs on the Keck I telescope.

TABLE 1

OBSERVING LOG

\begin{tabular}{llcc}
\hline \hline Telescope & \multicolumn{1}{c}{ Date } & Spectrograph & Imager \\
\hline Keck II...... & 1999 Sep 10 & $\ldots$ & LRIS + BVIfilters \\
CFHT ....... & 1999 Jul 14 & $\ldots$ & CFH12K + VR filters \\
Keck I ....... & 1998 Aug 21-22 & HIRES + C1 Decker & $\ldots$ \\
Keck I ....... & 1998 Oct 15-16 & HIRES + C5 Decker & $\ldots$ \\
Keck I ....... & 1999 Jul 15-16 & HIRES + C1 Decker & $\ldots$ \\
Keck I ........ & 1999 Aug 11 & HIRES + C1 Decker & $\ldots$ \\
\hline
\end{tabular}




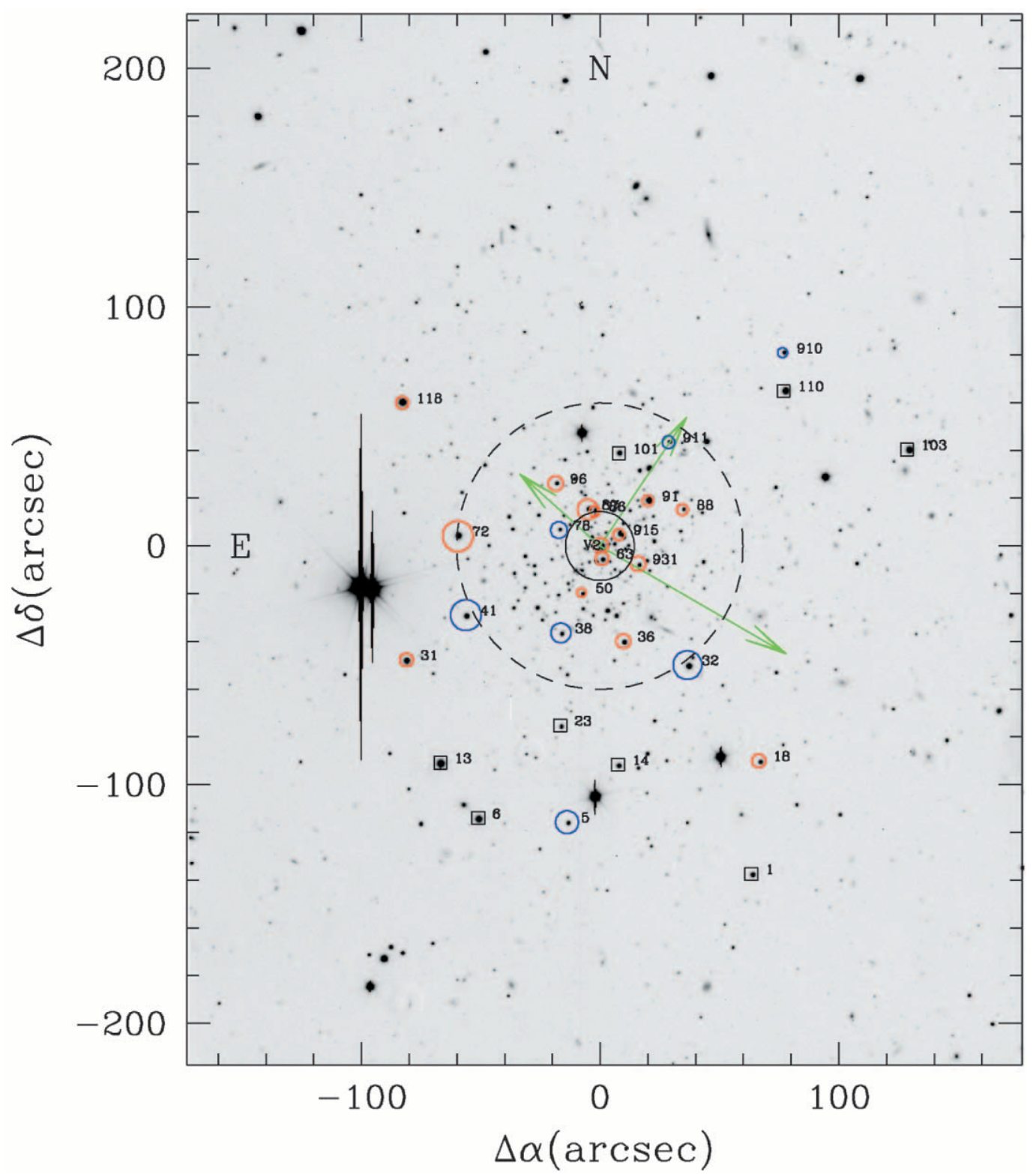

FIG. 1. $-V$-band image of Palomar 13 taken with LRIS on the Keck II telescope. This image measures $5 ! 8 \times 7 ! 3$. The solid circle shows the core radius $\left(r_{c}=14^{\prime \prime}\right)$ of the best-fit King-Michie model. The dashed circle denotes the "break" radius where the possible excess in the background-subtracted surface density profile begins: $R_{b} \simeq 1^{\prime}$ (see $\S 5.1$ ). The shortest of the three arrows shows the direction of the absolute proper motion from Siegel et al. (2001). The intermediate arrow points in the direction perpendicular to the Galactic plane, while the long arrow points in the direction of the Galactic center. Stars judged to be probable cluster members based on their radial velocities and proper motions are circled. The size of each circle is proportional to the absolute value of the difference in the measured radial velocity from the systemic velocity of the cluster. Red and blue circles denote stars with positive and negative velocity residuals, respectively. Nonmembers are indicated by open squares.

Candidate RGB stars in Palomar 13 were selected from previously published CMDs and finding charts (e.g., Ortolani et al. 1985 and Borissova et al. 1997). A few additional targets were identified on the basis of instrumental CMDs constructed from images obtained with COSMIC (Kells et al. 1998) on the Palomar $5 \mathrm{~m}$ telescope. During three of the four HIRES runs, we limited the entrance aperture to 0 ". $86 \times 7$ ". 0 with the $\mathrm{Cl}$ decker and binned the $2048 \times 2048$ detector $1 \times 2$ (i.e., in the spatial direction) to reduce the read noise. For the fourth run, we used the $\mathrm{C} 5$ decker (i.e., an entrance aperture of dimension 1 !' $15 \times 7$ ". 0 ) and binned the detector $2 \times 2$. The corresponding spectral resolutions for these two instrumental configurations are $\lambda / \Delta \lambda=45,000$ and 34,000 , respectively. In all cases, we used a single readout amplifier, a gain setting of $2.4 e^{-1}$ $\mathrm{ADU}^{-1}$, the red collimator and a cross-disperser in first order. The angles of the grating and cross disperser were adjusted to give complete spectral coverage over the range $5055 \AA \lesssim \lambda \lesssim 5355 \AA$. Thorium-Argon comparison lamp spectra were acquired frequently during each night- -usually before and after each program star observation. In a few cases, lamp spectra were separated by 30-60 minutes for short exposures of the brightest program stars. High $\mathrm{S} / \mathrm{N}$ spectra for 8-12 IAU radial velocity standard stars were obtained during each observing run.

All spectra (i.e., program objects and standard stars) were reduced in an identical manner following the general procedures described in earlier papers (e.g., Côté 1999; Côté et al. 

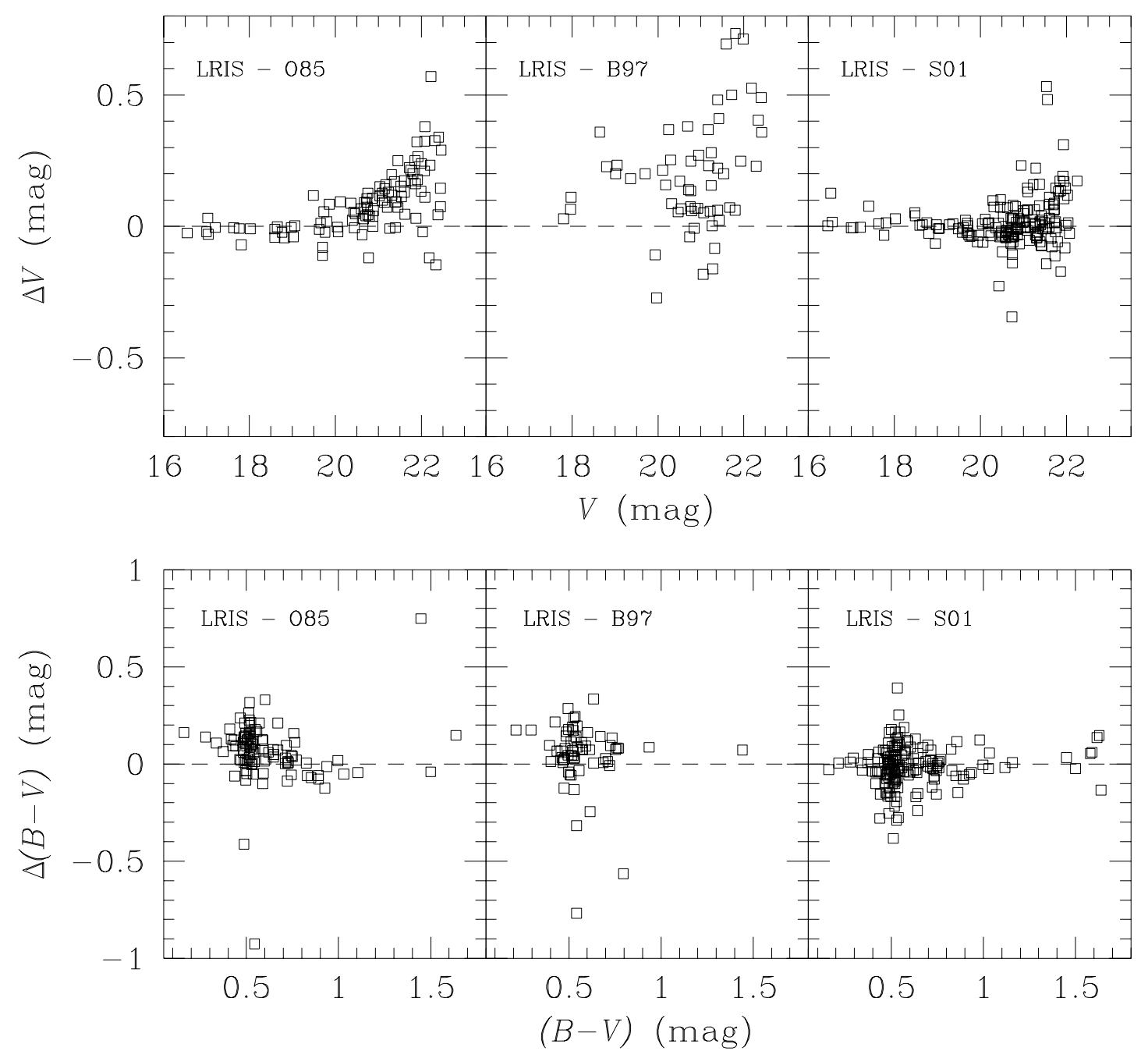

FIG. 2.-Top: Difference in $V$ magnitudes for stars in common between our LRIS photometric study and those of Ortolani et al. (1985; O85), Borissova et al. (1997; B97) and Siegel et al. (2001; S01). Bottom: Difference in $(B-V)$ color for stars in common between our LRIS photometric study and those of Ortolani et al. (1985; O85), Borissova et al. (1997; B97) and Siegel et al. (2001; S01).

1999). The radial velocity of each program object was measured by cross-correlating its spectrum against that of a master template created during each run from the observations of IAU standard stars. In order to minimize possible systematic effects, a master template for each observing run was derived from a similar, and in some cases identical, sample of IAU standard stars. From each cross-correlation function, we measured both $v_{r}$, the heliocentric radial velocity, and $R_{\mathrm{TD}}$, the Tonry \& Davis (1979) estimator of the strength of the cross-correlation peak. During the course of this program - which spanned seven observing runs totaling 13 nights - we obtained 53 distinct radial velocity measurements for 23 different program objects. All of these objects are faint, metal-poor members of our program clusters. Using the procedures described in Vogt et al. (1995) and Côté et al. (1999), we then derived empirical estimates for our radial velocity uncertainties. Specifically, we assume that the uncertainty of any radial velocity measurement, $\epsilon\left(v_{r}\right)$, can be expressed

$$
\epsilon\left(v_{r}\right)=\alpha /\left(1+R_{\mathrm{TD}}\right),
$$

where $\alpha$ is a constant to be determined. For our sample of repeat velocity measurements, which has 30 degrees of freedom, we calculate $\alpha=9.0_{-1.6}^{+2.4}$ for $\chi^{2}=1$, where the quoted uncertainties refer to $90 \%$ confidence limits.

Table 2 summarizes the results of our photometric and spectroscopic observations of Palomar 13. From left to right, columns (1)-(9) of this table record the name of each program star, ${ }^{5}$ distance from the cluster center, $V$ magnitude, $(B-V)$ color, HIRES exposure time, heliocentric Julian date, Tonry \& Davis $R_{\mathrm{TD}}$ value, heliocentric radial velocity, and the weighted mean velocity. Column (10) gives the cluster membership probability, $P(\mu)$, taken from the proper motion survey of Siegel et al. (2001). These membership probabilities are discussed in detail below. Column (11) gives our assessment of each star's membership in Palomar 13.

\footnotetext{
${ }^{5}$ Identifications are from Ortolani et al. (1985) for those stars beginning with either a "ORS" or "V" prefix; four additional stars $(910,911,915$ and 931) that were not included in the Ortolani et al. (1985) study were selected from images taken with COSMIC on the Palomar $5 \mathrm{~m}$ telescope.
} 
TABLE 2

Radial Velocities for Candidate Red Giants in Palomar 13

\begin{tabular}{|c|c|c|c|c|c|c|c|c|c|c|}
\hline $\begin{array}{l}\text { ID } \\
\text { (1) }\end{array}$ & $\begin{array}{c}R \\
(\operatorname{arcsec}) \\
(2)\end{array}$ & $\begin{array}{c}V \\
(\mathrm{mag}) \\
(3)\end{array}$ & $\begin{array}{c}(B-V) \\
(\mathrm{mag}) \\
(4)\end{array}$ & $\begin{array}{l}T \\
(\mathrm{~s}) \\
(5)\end{array}$ & $\begin{array}{c}\text { HJD } 2,450,000+ \\
(6)\end{array}$ & $\begin{array}{l}R_{\mathrm{TD}} \\
\text { (7) }\end{array}$ & $\begin{array}{c}v_{r} \\
\left(\mathrm{~km} \mathrm{~s}^{-1}\right) \\
(8)\end{array}$ & $\begin{array}{c}\left\langle v_{r}\right\rangle \\
\left(\mathrm{km} \mathrm{s}^{-1}\right) \\
(9)\end{array}$ & $\begin{array}{l}P(\mu) \\
(\%) \\
(10)\end{array}$ & $\begin{array}{c}\text { Member? } \\
\text { (11) }\end{array}$ \\
\hline ORS-13 ........ & 113 & 16.55 & 0.89 & $\begin{array}{l}180 \\
240\end{array}$ & $\begin{array}{l}1046.9063 \\
1047.9159\end{array}$ & $\begin{array}{l}17.61 \\
24.72\end{array}$ & $\begin{array}{l}-38.38 \pm 0.48 \\
-39.17 \pm 0.35\end{array}$ & $-38.90 \pm 0.29$ & 0 & $\mathrm{~N}$ \\
\hline ORS-118 ...... & 102 & 17.00 & 0.93 & $\begin{array}{l}270 \\
600 \\
720\end{array}$ & $\begin{array}{l}1046.9147 \\
1374.9741 \\
1375.9423\end{array}$ & $\begin{array}{l}13.04 \\
27.43 \\
29.27\end{array}$ & $\begin{array}{l}25.43 \pm 0.64 \\
25.07 \pm 0.32 \\
24.68 \pm 0.30\end{array}$ & $24.92 \pm 0.21$ & 0 & $\mathrm{Y}$ \\
\hline ORS-6 .......... & 125 & 17.03 & 1.10 & 240 & 1046.9104 & 18.00 & $0.68 \pm 0.47$ & $0.68 \pm 0.47$ & 0 & $\mathrm{~N}$ \\
\hline ORS-103 ...... & 134 & 17.04 & 1.00 & 360 & 1046.9196 & 24.48 & $-56.72 \pm 0.35$ & $-56.72 \pm 0.35$ & 0 & $\mathrm{~N}$ \\
\hline ORS-110 ...... & 100 & 17.21 & 1.03 & 600 & 1046.9266 & 23.96 & $5.40 \pm 0.36$ & $5.40 \pm 0.36$ & 0 & $\mathrm{~N}$ \\
\hline ORS-72 ....... & 60 & 17.64 & 0.85 & $\begin{array}{l}240 \\
750 \\
750 \\
900\end{array}$ & $\begin{array}{l}1101.7681 \\
1046.9350 \\
1047.9235 \\
1374.9973\end{array}$ & $\begin{array}{l}10.22 \\
13.45 \\
13.14 \\
24.38\end{array}$ & $\begin{array}{l}28.64 \pm 0.80 \\
29.30 \pm 0.62 \\
30.05 \pm 0.64 \\
28.26 \pm 0.36\end{array}$ & $28.79 \pm 0.27$ & 88 & $\mathrm{Y}$ \\
\hline ORS-31 ........ & 95 & 17.76 & 0.83 & $\begin{array}{l}750 \\
900\end{array}$ & $\begin{array}{l}1046.9456 \\
1374.9844\end{array}$ & $\begin{array}{l}10.70 \\
22.02\end{array}$ & $\begin{array}{l}26.31 \pm 0.77 \\
24.78 \pm 0.39\end{array}$ & $25.09 \pm 0.35$ & 79 & $\mathrm{Y}$ \\
\hline ORS-91 ........ & 27 & 17.81 & 0.67 & $\begin{array}{l}750 \\
900\end{array}$ & $\begin{array}{l}1046.9554 \\
1375.0091\end{array}$ & $\begin{array}{r}6.72 \\
12.39\end{array}$ & $\begin{array}{l}24.46 \pm 1.17 \\
24.64 \pm 0.67\end{array}$ & $24.59 \pm 0.60$ & 99 & $\mathrm{Y}$ \\
\hline $\mathrm{V} 2 \ldots \ldots \ldots \ldots \ldots$ & 0 & 17.91 & 0.49 & 1200 & 1375.1025 & 9.32 & $25.39 \pm 0.87$ & $25.39 \pm 0.87$ & $\ldots$ & $\mathrm{Y}$ \\
\hline ORS-32 ........ & 62 & 18.02 & 0.89 & $\begin{array}{r}900 \\
900 \\
1200 \\
1200\end{array}$ & $\begin{array}{l}1046.9664 \\
1375.1170 \\
1376.1122 \\
1401.9353\end{array}$ & $\begin{array}{l}11.56 \\
18.95 \\
18.60 \\
17.23\end{array}$ & $\begin{array}{l}20.46 \pm 0.72 \\
19.31 \pm 0.45 \\
18.69 \pm 0.46 \\
20.90 \pm 0.49\end{array}$ & $19.68 \pm 0.26$ & 35 & $\mathrm{Y}$ \\
\hline d-41................. & 64 & 18.59 & 0.76 & $\begin{array}{r}320 \\
1200 \\
1500\end{array}$ & $\begin{array}{l}1101.7397 \\
1046.9987 \\
1375.9567\end{array}$ & $\begin{array}{r}3.91 \\
8.72 \\
15.56\end{array}$ & $\begin{array}{l}18.37 \pm 1.84 \\
18.91 \pm 0.93 \\
19.43 \pm 0.54\end{array}$ & $19.24 \pm 0.47$ & 88 & $\mathrm{Y}$ \\
\hline ORS-1 $\ldots \ldots \ldots$ & 151 & 18.59 & 0.82 & 1200 & 1046.9799 & 13.35 & $-37.48 \pm 0.63$ & $-37.48 \pm 0.63$ & 0 & $\mathrm{~N}$ \\
\hline ORS-101 ...... & 39 & 18.65 & 0.94 & 1200 & 1047.0141 & 18.88 & $-17.39 \pm 0.45$ & $-17.39 \pm 0.45$ & 0 & $\mathrm{~N}$ \\
\hline ORS-14 ........ & 92 & 18.76 & 0.74 & 1500 & 1047.0337 & 17.63 & $-55.31 \pm 0.48$ & $-55.31 \pm 0.48$ & 0 & $\mathrm{~N}$ \\
\hline ORS-86 ........ & 14 & 18.81 & 0.77 & 1800 & 1047.8735 & 10.01 & $24.47 \pm 0.82$ & $24.47 \pm 0.82$ & 99 & $\mathrm{Y}$ \\
\hline ORS-36 ........ & 41 & 18.98 & 0.75 & 1800 & 1047.0760 & 9.13 & $25.29 \pm 0.89$ & $25.29 \pm 0.89$ & 93 & $\mathrm{Y}$ \\
\hline ORS-63 ......... & 6 & 19.02 & 0.76 & $\begin{array}{l}1800 \\
2100\end{array}$ & $\begin{array}{l}1047.0530 \\
1375.0805\end{array}$ & $\begin{array}{r}9.12 \\
18.35\end{array}$ & $\begin{array}{l}26.09 \pm 0.89 \\
24.82 \pm 0.47\end{array}$ & $25.09 \pm 0.42$ & 62 & $\mathrm{Y}$ \\
\hline ORS-87 ........ & 16 & 19.05 & 0.72 & 1800 & 1047.9021 & 9.06 & $26.55 \pm 0.90$ & $26.55 \pm 0.90$ & 94 & $\mathrm{Y}$ \\
\hline $910 \ldots \ldots \ldots \ldots \ldots$ & 111 & 19.28 & 0.73 & 600 & 1101.7614 & 4.15 & $23.77 \pm 1.75$ & $23.77 \pm 1.75$ & 89 & $\mathrm{Y}$ \\
\hline $911 \ldots \ldots \ldots \ldots \ldots$ & 52 & 19.36 & 0.75 & 600 & 1101.7306 & 3.86 & $23.19 \pm 1.86$ & $23.19 \pm 1.86$ & 99 & $\mathrm{Y}$ \\
\hline ORS-23 ........ & 77 & 19.49 & 1.50 & 2400 & 1047.1068 & 10.01 & $18.57 \pm 0.82$ & $18.57 \pm 0.82$ & 0 & $\mathrm{~N}$ \\
\hline $915 \ldots \ldots \ldots \ldots \ldots$ & 9 & 19.58 & 0.73 & 1800 & 1375.0284 & 11.68 & $24.69 \pm 0.71$ & $24.69 \pm 0.71$ & $\ldots$ & $\mathrm{Y}$ \\
\hline ORS-18 ........ & 112 & 19.62 & 0.73 & 1000 & 1102.7253 & 4.36 & $25.16 \pm 1.68$ & $25.16 \pm 1.68$ & 98 & $\mathrm{Y}$ \\
\hline ORS-38 ........ & 40 & 19.65 & 0.74 & $\begin{array}{l}1000 \\
2400\end{array}$ & $\begin{array}{l}1101.7489 \\
1376.0856\end{array}$ & $\begin{array}{r}4.49 \\
10.57\end{array}$ & $\begin{array}{l}19.52 \pm 1.64 \\
22.11 \pm 0.78\end{array}$ & $21.64 \pm 0.72$ & 88 & $\mathrm{Y}$ \\
\hline 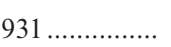 & 18 & 19.70 & 0.72 & 2100 & 1375.0528 & 11.70 & $25.45 \pm 0.71$ & $25.45 \pm 0.71$ & 91 & $\mathrm{Y}$ \\
\hline ORS-78 ......... & 19 & 19.73 & 0.72 & 1000 & 1102.7404 & 5.26 & $22.34 \pm 1.44$ & $22.34 \pm 1.44$ & 78 & $\mathrm{Y}$ \\
\hline ORS-50 ........ & 22 & 19.76 & 0.72 & 2700 & 1376.0226 & 9.66 & $24.15 \pm 0.85$ & $24.15 \pm 0.85$ & 98 & $\mathrm{Y}$ \\
\hline ORS-96 ........ & 32 & 19.80 & 0.71 & 1000 & 1102.7542 & 6.52 & $25.38 \pm 1.20$ & $25.38 \pm 1.20$ & 99 & $\mathrm{Y}$ \\
\hline ORS-5 .......... & 117 & 19.86 & 0.72 & 2700 & 1375.9867 & 9.99 & $20.95 \pm 0.82$ & $20.95 \pm 0.82$ & 98 & $\mathrm{Y}$ \\
\hline ORS- $88 \ldots \ldots \ldots$ & 37 & 20.11 & 0.71 & 2100 & 1376.0541 & 7.34 & $24.61 \pm 1.08$ & $24.61 \pm 1.08$ & 78 & $\mathrm{Y}$ \\
\hline
\end{tabular}

\section{RESULTS}

\subsection{Identification of Members and Nonmembers}

A crucial first step in studying the internal dynamics of Palomar 13 is the isolation of a sample of bona fide members. At a Galactic latitude of $b=-42.7$, Palomar 13 is located well below the Galactic plane, but its very sparse giant branch ${ }^{6}$ means that contamination from foreground disk and halo stars may be nonnegligible. As a further complication, there is only limited radial velocity separation with the disk field star population due to Palomar 13's low systemic velocity (see $\S 3.5$ ). These difficulties are amelio-

${ }^{6}$ Ortolani et al. (1985) reported that the cluster luminosity function is similar to that of $\mathrm{M} 3$, but reduced by a factor of $\sim 1 / 60$. rated by the high precision of our radial velocity measurements, our new CMD, and the availability of proper motions from Siegel et al. (2001).

In Figure 3, we plot the mean radial velocities of our program stars, $\left\langle v_{r}\right\rangle$, against membership probability, $P(\mu)$, from Siegel et al. (2001). ${ }^{7}$ The sharp spike at $\left\langle v_{r}\right\rangle \sim 25 \mathrm{~km}$

${ }^{7}$ Two stars have been omitted: V2 (a known RR Lyrae; Ciatti, Rosino, $\&$ Sussi 1965) and 915. Siegel et al. (2001) were unable to measure proper motions for either of these two objects, but both stars are almost certainly members based on their radial velocities, their location in CMDs, and their proximity to the center of Palomar 13 (see Table 2, col. [2], and Fig. 1). Nevertheless, V2 has been excluded from the calculation of the observed velocity dispersion since atmospheric pulsations may inflate the measured dispersion (e.g., the peak-to-peak radial velocity variations of RR Lyrae stars in M92 are $\Delta v_{r}=50-60 \mathrm{~km} \mathrm{~s}^{-1}$; Cohen 1992). 


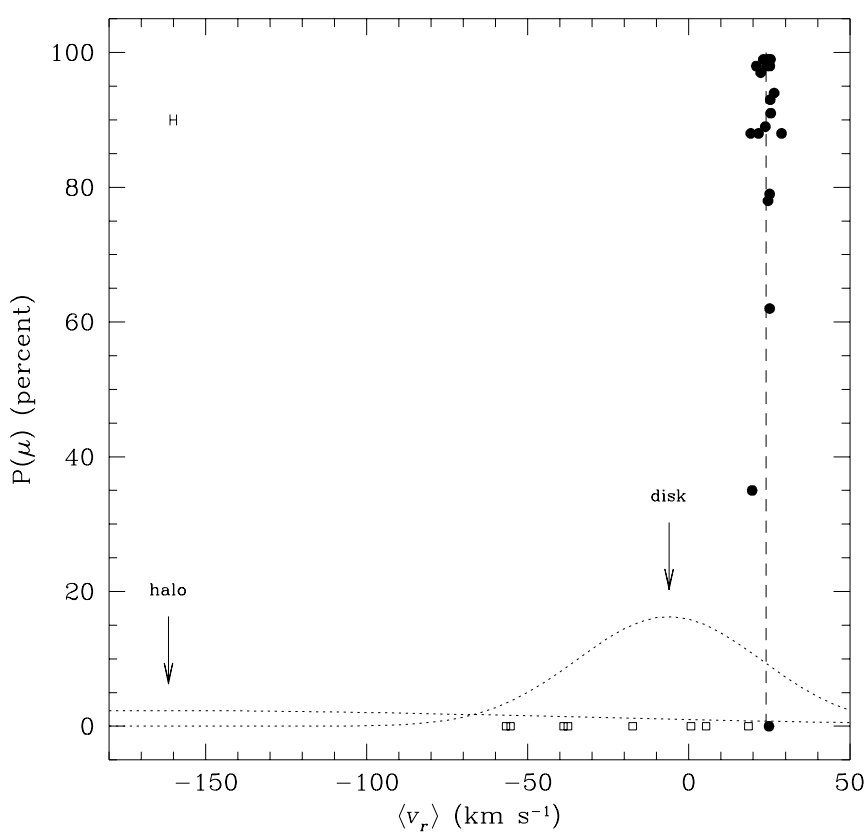

FIG. 3.-Proper motion membership probability from Siegel et al. (2001), plotted against mean radial velocity for candidate Palomar 13 stars. The dotted curves show approximate velocity distributions along this line of sight for disk and halo field stars (see text for details). The dashed vertical line indicates the systemic velocity of Palomar 13. The error bar in the upper left corner shows the typical uncertainty in the measured radial velocity. Open squares show non-members, while probable cluster members are indicated by the filled circles. Note that this latter sample includes one star (ORS-118) that has $P(\mu)=0 \%$ according to Siegel et al. (2001). We argue that this star is, in fact, a cluster member since it has a radial velocity that is indistinguishable from cluster systemic velocity, it is located precisely on the red giant branch in the cluster color-magnitude diagram, and it has a metallicity of $[\mathrm{Fe} / \mathrm{H}]=-1.98 \pm 0.31$ dex measured from our HIRES spectra.

$\mathrm{s}^{-1}$ identifies Palomar 13; the vertical dashed line indicates our best estimate for its systemic velocity (see $\S 3.5$ ). In general, there is good agreement between the membership classifications based on the radial velocities and those based on the proper motions. The three exceptions are ORS-23, ORS-32, and ORS-118. In the first case, the measured radial velocity of $v_{r}=18.57 \pm 0.82 \mathrm{~km} \mathrm{~s}^{-1}$ for ORS-23 provides no compelling evidence either for, or against, membership. However, Siegel et al. (2001) find $P(\mu)=0 \%$ for this star, a conclusion that is supported by the location of this star in the CMD; i.e., the star has a very red color of $(B-V)=1.50$ mag and is located $\sim 0.8 \mathrm{mag}$ blueward of Palomar 13's RGB. We conclude that ORS-23 is a likely field star. For ORS-32, Siegel et al. (2001) report an intermediate probability of $P(\mu)=35 \%$. Our mean radial velocity, $v_{r}=19.68 \pm 0.26 \mathrm{~km} \mathrm{~s}^{-1}$, is based on four measurements at three different epochs. The residual with respect to the systemic velocity of Palomar 13 is $4.5 \mathrm{~km} \mathrm{~s}^{-1}$, and the star is located $R \sim 1^{\prime}$ from the core of Palomar 13. We believe that these facts, coupled with the position of this star in the $\mathrm{CMD}$, marginally favor the interpretation that it is a member of Palomar 13. Nevertheless, a spectroscopic measurement of the metallicity of this star would be desirable.

The situation for ORS-118 is somewhat ambiguous. Although Siegel et al. (2001) report $P(\mu)=0 \%$ for this star, its location in the CMD strongly suggests that it is a true member, since it lies precisely on the RGB of the best-fit iso- chrone (see $\S 3.3$ ). The measured radial velocity of this star, $v_{r}=24.92 \pm 0.21 \mathrm{~km} \mathrm{~s}^{-1}$, is indistinguishable from the systemic velocity of Palomar 13 and is based on three independent measurements that agree internally to better than $0.75 \mathrm{~km} \mathrm{~s}^{-1}$. We further note that an abundance analysis for ORS-118 (described below) yields $[\mathrm{Fe} / \mathrm{H}]=-1.98 \pm 0.31$ dex. This metallicity points to a halo origin and is consistent with the metallicity of Palomar 13 deduced from isochrone fitting (see $\S 3.3$ ).

To investigate the possibility that ORS-118 is simply an interloping foreground star that happens to have the same radial velocity as the Palomar 13, we approximate the lineof-sight velocity distributions expected for disk and halo stars in this direction with the relation

$$
P\left(v_{r}\right) \propto \exp \left[-\left(\left\langle v_{r}\right\rangle-\left\langle v_{r, 0}\right\rangle\right)^{2} / 2 \sigma^{2}\right],
$$

where

$$
\left\langle v_{r, 0}\right\rangle=-19.5 \cos \phi_{d}
$$

is the mean radial velocity of disk stars along this line of sight as a result of the solar motion toward $l=56^{\circ}$ and $b=23^{\circ}$ (Mihalas \& Binney 1981). The angle between this point on the celestial sphere and Palomar 13 is denoted by $\phi_{d}$. Similarly, the mean radial velocity of halo stars along this line of sight is taken to be

$$
\left\langle v_{r, 0}\right\rangle=-220 \cos \phi_{h},
$$

where $\phi_{h}$ is the angle subtended by Palomar 13 and $(l, b)=\left(90^{\circ}, 0^{\circ}\right)$. We further approximate the local velocity dispersion of disk stars as

$$
\sigma^{2} \simeq \frac{1}{3}\left(\sigma_{U}^{2}+\sigma_{Y}^{2}+\sigma_{Z}^{2}\right)
$$

which, from the last row of Table 1 of Dehnen \& Binney (1998), yields $\sigma \simeq 29 \mathrm{~km} \mathrm{~s}^{-1}$. For the halo, we assume an isotropic velocity ellipsoid (i.e., $\sigma_{U}=\sigma_{V}=\sigma_{Z}$ ) and adopt $\sigma \simeq 124 \mathrm{~km} \mathrm{~s}^{-1}$ from Côté (1999).

The resulting probability distributions for disk and halo stars are illustrated by the dotted lines in Figure 3. Although the absolute normalization of the two curves is arbitrary, their relative normalization has been adjusted to match the expected number of disk and halo stars with $16.5 \leq V \leq 20$ along this line of sight (determined from the IAS Galaxy model; Bahcall \& Soneira 1980). If ORS-118 is indeed a halo field star, then it lies $1.5 \sigma$ from the mean of the field distribution; the probability of such an occurrence is $13 \%$. Moreover, in the range $16.5 \leq V \leq 17.5$, the IAS Galaxy model predicts only a single halo field star in our field, and just $8 \%$ of halo stars in this magnitude range are expected to have $(B-V) \geq 0.93$ (i.e., the color of ORS-118). On the basis of these considerations, we conclude that ORS-118 is likely to be a bona fide member of Palomar 13. The origin of the large proper motion residual measured by Siegel et al. (2001) is unclear, particularly since an inspection of our $\mathrm{CCD}$ images revealed no close companions.

\subsection{Metallicity}

Exposure times for our HIRES observations were chosen to yield the minimum $\mathrm{S} / \mathrm{N}$ needed to derive radial velocities with a precision $\epsilon\left(v_{r}\right) \lesssim 1 \mathrm{~km} \mathrm{~s}^{-1}$. For ORS-118, the brightest star in our sample that has a radial velocity consistent with membership in Palomar 13, we combined the two 
spectra obtained during the 1999 July observing run. The $\mathrm{S} / \mathrm{N}$ of this co-added spectrum- $\mathrm{S} / \mathrm{N} \simeq 25$ per resolution element - is just adequate for abundance analysis.

Our analysis used $28 \mathrm{Fe}$ I lines with oscillator strength values adopted from McWilliam et al. (1995), Kurucz model atmospheres, and solar abundances from Anders \& Grevesse (1989). Computations were made with the most recent version of the LTE line analysis code MOOG (Sneden 1973). The effective temperature, $T_{e}$, was first determined using an initial estimate from the photometry and then checked through excitation equilibrium of the $\mathrm{Fe}$ I lines whose equivalent widths were measured using standard IRAF routines. The surface gravity, $\log g$, was derived from the ionization equilibrium of $\mathrm{Fe}$ I and $\mathrm{Fe}$ II lines using four $\mathrm{Fe}$ II lines in the available spectral region. Once the effective temperature and surface gravity were determined, abundances for the Fe I lines were calculated by iteratively varying the microturbulent velocity until the best fit in the diagram of $\mathrm{Fe}$ I abundance versus equivalent width was achieved. Our best-fit parameters for ORS-118 are $T_{e}=4700 \mathrm{~K}$, $\log g=1.7$, and $[\mathrm{Fe} / \mathrm{H}]=-1.98 \pm 0.31 \mathrm{dex}$, where the rather large uncertainty on the measured metallicity is a reflection of the modest $\mathrm{S} / \mathrm{N}$ of our co-added spectrum.
Previous estimates for the metallicity of Palomar 13 include $[\mathrm{Fe} / \mathrm{H}]=-1.9 \pm 0.4$ (Canterna \& Schommer 1978), $-1.67 \pm 0.15$ (Zinn \& Diaz 1982), and $-1.9 \pm 0.1 \mathrm{dex}$ (Friel et al. 1982). The measurement of Canterna \& Schommer (1978) comes from Washington photometry of individual stars, while the latter two measurements are based on lowresolution spectroscopy. Our determination of the cluster metallicity is slightly lower than, but still consistent with, these previous estimates. In the following section, we show that this metallicity is also consistent with that found from isochrone fitting of the CMD.

\subsection{Color-Magnitude Diagram}

Figure 4 shows $B V$ and $V I$ CMDs for Palomar 13 constructed from our LRIS photometry. As noted by previous investigators, the RGB is very sparsely populated. This figure (and Fig. 6, left panel) also confirms the finding of Siegel et al. (2001) that Palomar 13 contains a population of blue stragglers; likewise, there may be evidence for a "second sequence" that runs parallel to the upper main sequence. Both this latter sequence (which is traditionally interpreted as the signature of unresolved binary stars; e.g., Romani \& Weinberg 1991) and the presence of blue stragglers (a

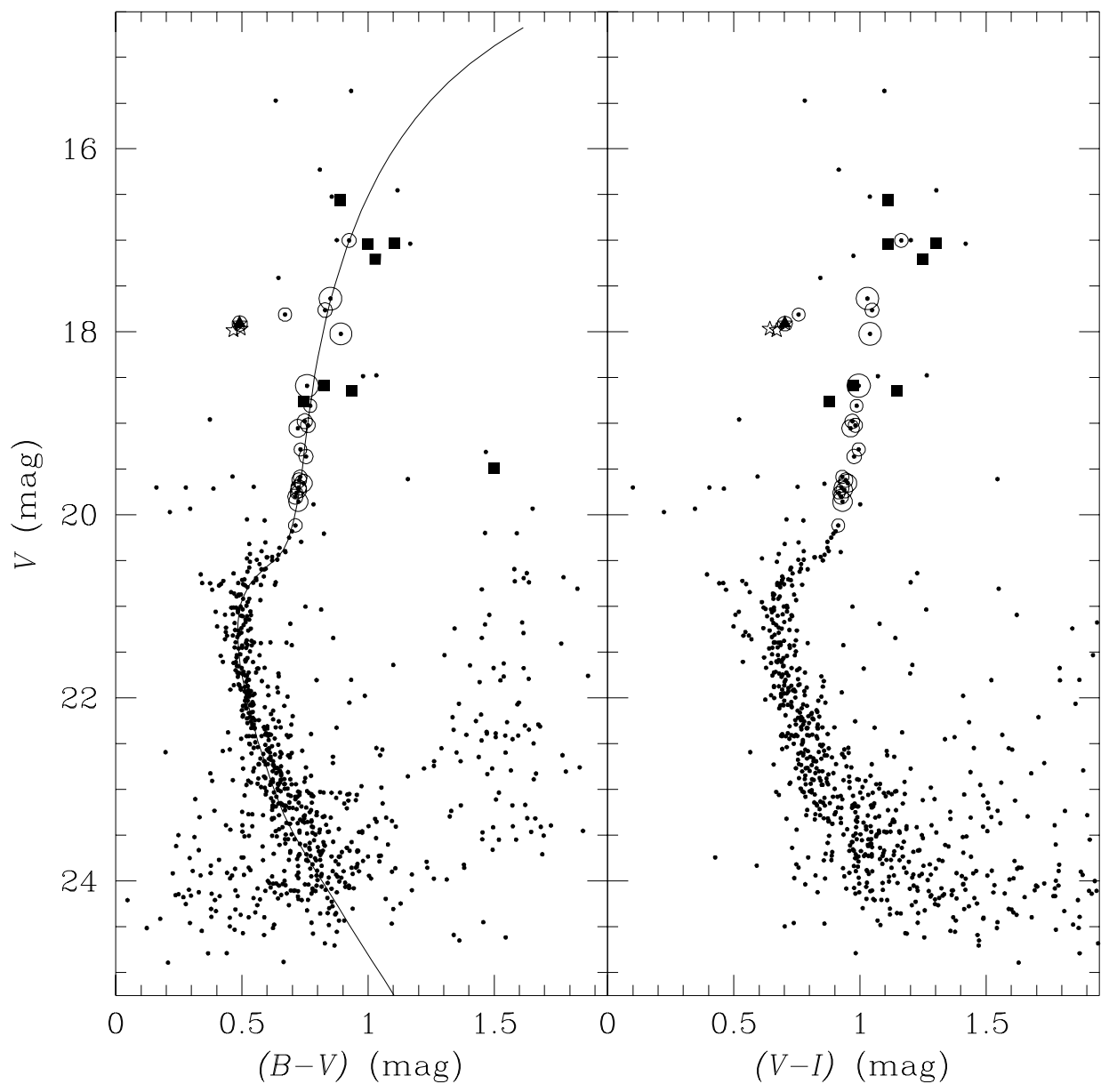

FIG. 4.-Left: $V,(B-V)$ color-magnitude diagram for Palomar 13 based on our LRIS images. A total of 840 objects are plotted in this figure. Open circles indicate probable cluster members, determined from our HIRES radial velocities. The size of each circle is proportional to the absolute value of velocity residual with respect to the cluster's systemic velocity. Radial velocity non-member stars are indicated by the filled squares. Known RR Lyrae variables are indicated by the stars; the circled variable is V2. The solid curve shows an isochrone from Bergbusch \& VandenBerg (1992) having an age of T $=14 \mathrm{Gyr}$ and a metallicity $[\mathrm{Fe} / \mathrm{H}]=-1.78$, which has been shifted by $E(B-V)=0.11 \mathrm{mag}$ (Schlegel et al. 1998) and $(m-M)_{V}=17.27 \mathrm{mag}$. Right: $V,(V-I)$ colormagnitude diagram for Palomar 13 derived from our LRIS images. The symbols are the same as in the previous panel. 
fraction of which are likely to be W UMa systems; Mateo et al. 1990) provide some evidence for a population of binary stars. If this is indeed the case, then it would have important implications for modeling the observed velocity dispersion profile. In $\S 5.1$, we shall return to the question of binary stars and their possible effect on the observed velocity dispersion.

From the DIRBE maps of Schlegel, Finkbeiner, \& Davis (1998), we find the reddening in the direction of Palomar 13 to be $E(B-V)=0.11 \mathrm{mag}$. The solid curve in the left panel of Figure 4 shows a $14 \mathrm{Gyr}$ isochrone from Bergbusch \& VandenBerg (1992) with $[\mathrm{Fe} / \mathrm{H}]=-1.78 \mathrm{dex}$, shifted by $(m-M)_{V}=17.27 \mathrm{mag}$. We stress that the sparsely populated red giant and horizontal branches preclude a precise age determination and simply note that a $14 \mathrm{Gyr}$ isochrone provides a reasonable match to the observed fiducial sequences. Assuming $A_{V}=3.1 E(B-V)$, we find a true distance modulus of $(m-M)_{0}=16.93 \pm 0.10$ mag, which corresponds to a distance of $D=24.3_{-1.1}^{+1.2} \mathrm{kpc}$. This value is in excellent agreement with the distance of $D=24.8$ estimated by Siegel et al. (2001) from the average magnitude of a small sample of cluster RR Lyrae and horizontal branch stars. On the basis of the combination of isochrone fitting to the cluster CMD and the spectroscopic analysis of ORS-118, we adopt $[\mathrm{Fe} / \mathrm{H}]=-1.9 \pm 0.2$ dex as our best estimate for the metallicity of Palomar 13.

Ortolani et al. (1985) found an absolute magnitude of $M_{V}=-3.4$ mag for Palomar 13 by integrating the luminosity function. For a distance of $D=24.3$, we find an integrated luminosity of $2.4 \times 10^{3} L_{V} \odot\left(M_{V}=-3.6\right)$ within the tidal radius suggested by Siegel et al. (2001), $r_{t} \simeq 188^{\prime \prime}$ (however, as discussed in $\S 3.4$, we see no evidence for a tidal cutoff at this, or any other, radius). This estimate excludes stars with $(B-V)<1.3 \mathrm{mag}$ and confirmed nonmembers. If the integration is extended over the entire LRIS field of view (as would be appropriate for the larger tidal radius found in $\S 4)$, the luminosity increases to $3.1 \times 10^{3} L_{V}$. $\left(M_{V}=-3.9\right)$. Our corresponding best estimate for the absolute magnitude of Palomar 13 is $M_{V}=-3.8 \mathrm{mag}$ (see also $\S 4-5)$. In the most recent version of the Harris (1996) catalog of Galactic globular clusters, only three objects-E 3, Palomar 1, and AM 4 -have lower luminosities. ${ }^{8}$ The integrated, dereddened color is $\langle B-V\rangle_{0}=0.65 \pm 0.04 \mathrm{mag}$, roughly consistent with that expected for a globular cluster of metallicity $[\mathrm{Fe} / \mathrm{H}] \simeq-1.9 \operatorname{dex}($ Durrell et al. 1996).

\subsection{Surface Density and Surface Brightness Profiles}

While there are surprisingly few published studies of the structural properties of Palomar 13, previous results have consistently pointed to a compact, faint cluster of low concentration. For instance, Webbink (1985) quotes a core radius of $r_{c}=23^{\prime \prime}$ and a concentration index of $c \equiv \log \left(r_{t} / r_{c}\right)=0.9$, while Trager, King, \& Djorgovski (1995) report $r_{c}=29^{\prime \prime}$ and $c=0.66$. However, both of these studies relied primarily on the star counts of Kinman \& Rosino (1962), which were obtained by viewing a photographic plate through a mask fixed on the projection screen of a Sartorious astrophotometer. Recently, Siegel et al. (2001) adopted $c=0.7$ and measured $r_{c}=39^{\prime \prime}$ for Palomar

\footnotetext{
${ }^{8}$ According to Harris (1996), the absolute magnitude of Terzan 1 is $M_{V}=-3.3$, i.e., fainter than Palomar 13. However, Idiart et al. (2001) have recently reported an upward revision to $M_{V} \simeq-5.4$ for this cluster.
}

13 using a sample of 119 members selected on the basis of their proper motions. They noted, however, that this model provides a poor description of the measured profile at large radii; i.e., 14 of their proper motion members $(12 \%$ of the sample) lay beyond the canonical tidal radius. This fact, coupled with Palomar 13's low luminosity and eccentric orbit, led Siegel et al. (2001) to suggest that this object is in the final stages of destruction.

We have used our Keck and CFHT imaging to measure improved structural parameters for Palomar 13. The pointsource catalog from our Keck images consists of 840 objects distributed over an area of $42.8 \mathrm{arcmin}^{2}$. Since the Palomar 13 field contains a significant number of obvious foreground/background objects (see Fig. 4), the Keck pointsource catalog was trimmed to exclude objects with $0.0 \mathrm{mag}<(B-V)<1.3 \mathrm{mag}$. Similarly, sources that did not fall in the range $0.15<(V-r)<0.7$ were culled from the CFHT catalog. The point-source catalogs were further restricted to objects brighter than $V=24$ (Keck) and 23.5 (CFHT) in order to ensure photometric completeness at all radii. This leaves a total of 629 and 551 objects in the Keck and CFHT databases, respectively.

Figure 5 shows the surface density profiles determined from these catalogs. Observations from Keck are shown as open squares, while open circles denote CFHT data points. The latter have been scaled upward by a factor $(629 / 551) \simeq$

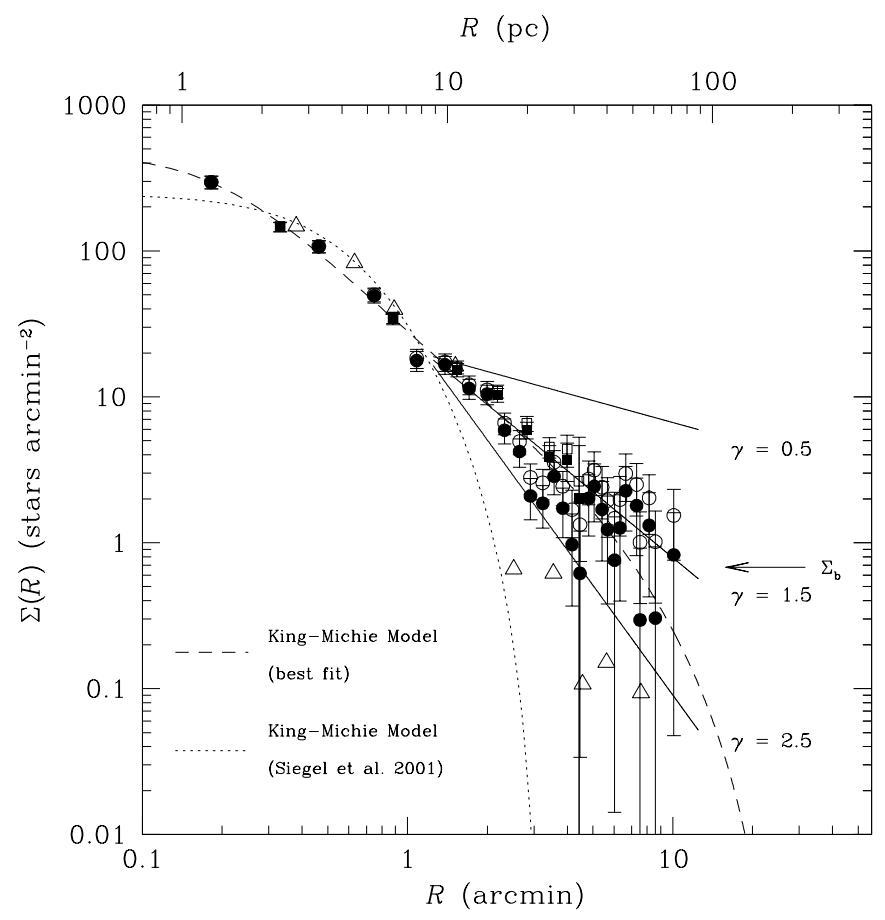

FIG. 5.-Surface density profile for Palomar 13 based on our Keck and CFHT photometry (squares and circles, respectively). Open symbols show the uncorrected surface density profile; filled symbols show the profile found after correcting the raw star counts for background contamination. For comparison, the surface density profile of Siegel et al. (2001) is illustrated by the open triangles, after scaling their counts upward by a factor of $(629 / 119) \simeq 5.29$ (i.e., the ratio of the number of stars used to derive the respective profiles). The dotted curve shows a King model with concentration parameter $c=0.7$ and core radius $r_{c}=39^{\prime \prime}$ suggested by Siegel et al. (2001). The dashed curve shows the formal best-fit King-Michie model. The three dashed lines beginning at $R \simeq 1^{\prime}$ indicate profiles of the form predicted by Johnston et al (2001) for extra-tidal stars: $\Sigma \propto R^{-\gamma}$ with $\gamma=0.5,1.5$, and 2.5 
1.14 to account for the difference in sample size. There is good agreement between these two independent profiles. Given the larger radial extent of the CFHT data, they provide stronger constraints on the asymptotic behavior of the surface density profile - a particularly important issue in the present case since the Keck surface density profile continues to decline out to the edge of the LRIS field of view. The arrow in Figure 5 shows our best estimate for the mean background surface density, $\Sigma_{b}=0.68$ stars $\operatorname{arcmin}^{-2}$. For comparison, the surface density of Galactic stars in the appropriate color and magnitude intervals in the direction of Palomar 13 predicted by Institute for Advanced Study Galaxy model (Bahcall \& Soneira 1980; Bahcall 1986) fall in

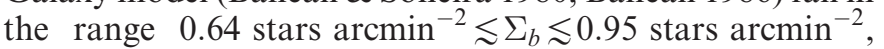
consistent with our empirical estimate. The filled symbols in this figure show the background-subtracted surface density profile.

For comparison, the open triangles in Figure 5 shown the surface density profile of Siegel et al. (2001), taken directly from a digitized image of their Figure 7. The Siegel et al. data points have been scaled upward by the ratio of the numbers of stars in the two studies: $(629 / 119) \simeq 5.29$. In the inner regions of Palomar 13, there is good agreement between the two profiles. Beyond a distance of $R=2^{\prime}-3^{\prime}$, however, the profiles differ, with our surface densities being higher by a factor of $\sim 5$ (however, to within the rather large uncertainties, the slopes of the two profiles are in agreement). The origin of this difference is unclear. The profile of Siegel et al. (2001) has the obvious advantage of being proper motion selected; i.e., objects having membership probabilities of $P(\mu)<50 \%$ have been rejected on a star-bystar basis. In principal, the use of proper motions to cull interlopers should be superior to the statistical approach used here to estimate the background level. On the other hand, Siegel et al. (2001) note that most of their plates extend to only $V \sim 21$ (i.e., the approximate location of the main-sequence turnoff), whereas each of our two independent sets of CCD photometry reach several magnitudes below this level and with small photometric errors. If Palomar 13 is experiencing significant mass loss through evaporation and tidal stripping (see $\S 5.2$ ), then it is the lowest mass stars that are expected to have the largest evaporation rates (i.e., they should preferentially inhibit the cluster envelope as a result of mass segregation). In such a case, our deeper star counts compared to Siegel et al. (2001) might lead to a more pronounced surface density excess at large radii. In any event, we caution that the five outermost bins in the Siegel et al. (2001) profile (spanning the range $2 \lesssim R \lesssim 10$ ) contain just 15 stars, so it is unclear of the difference between the two studies is truly significant.

With these caveats in mind, we point out a striking feature of Figure 5-the near power-law behavior of the surface density profile. Indeed, a single power law, $\Sigma(R) \propto R^{-\gamma}$, with $\gamma=1.8 \pm 0.2$ provides an adequate description of the surface density profile for $R \gtrsim 0 ! 3$. The dotted curve in Figure 5 shows the King-Michie model proposed by Siegel et al. (2001). This model, which has a somewhat arbitrary concentration parameter of $c=0.7$ and a core radius of $r_{c}=39^{\prime \prime}$, provides adequate description of the observed surface density profile over the range $0.3 \lesssim R \lesssim 1$ but underestimates the central surface density. More importantly, if this model accurately reflects the true density profile of Palomar 13, there is an even more dramatic "excess" of stars above the model predictions beyond $R \gtrsim 1$. While it is certainly true that the exact shape of the surface density profile depends on the adopted background level (and particularly so at large radii), this excess is unlikely to be simply an artifact of the background subtraction. For instance, achieving an acceptable fit to a King model with $c \lesssim 1$ for $R \lesssim 2$ would require the background level to be increased by roughly an order of magnitude.

The dashed curve in Figure 5 shows the best-fit KingMichie model, which will be discussed in detail in $\S 4$. As shown there, the King-Michie model that best fits the observed surface density and surface brightness profiles of Palomar 13 has a tidal radius of $r_{t}=26^{\prime} \pm 6^{\prime}(182 \pm 41 \mathrm{pc})$. Since this is more than twice the radial extent of our CFHT imaging, the adopted background level should be viewed with some caution. However, we note that estimating the background surface density from star counts made within $r_{t}$ should result in an overestimate of the true background level and, hence, to an underestimate of $r_{t}$. We are therefore left with two possible interpretations of the surface density profile of Palomar 13: either this object contains a significant population of "extra-tidal" stars that have been caught in the act of evaporation (as suggested by Siegel et al. 2001), or its concentration and spatial extent have been greatly underestimated in previous studies.

We now turn our attention to the properties of the stars associated with this surface density excess. This feature is evident in both the CFHT and LRIS surface density profiles, but is there any evidence that this population is truly comprised of Palomar 13 members? Figure 6 shows instrumental CMDs for the Palomar 13 field derived from our CFHT photometry, along with the color and magnitude limits used to cull probable nonmembers from the sample (i.e., the dotted regions). The panel on the left shows the CMD for all stars within the Siegel et al. (2001) tidal radius. The middle panel shows the CMD for all objects beyond this radius, while the right panel shows those stars located at more than twice this distance. The dashed curves in the final two panels show the Palomar 13 fiducial sequence. A significant number of probable members with $R>r_{t}$ are evident, providing additional evidence that most of the stars belonging to the excess in the surface density profile are indeed associated with Palomar 13.

Figure 7 shows the surface brightness profile for Palomar 13 measured from our Keck and CFHT images. Profiles having been calculated independently using both the Keck and CFHT photometry. Surface photometry is shown as the pentagons (Keck) and triangles (CFHT), while the results from star counts are indicated by the squares (Keck) and circles (CFHT). A direct measurement of the background surface brightness for the Keck and CFHT images yields $\mu_{V}=21.3$ and $21.5 \mathrm{mag} \operatorname{arcsec}^{-2}$, respectively-consistent with expectations for dark sky conditions at Mauna Kea during 1999 (Krisciunas 1997). Note that in deriving a surface brightness profile from the star counts discussed above, we have simply multiplied the surface densities shown in Figure 5 by a constant factor, $\eta=L_{V} / n_{\text {tot }}$, where $L_{V}$ is the integrated luminosity of Palomar 13 and $n_{\text {tot }}$ are the number of stars used to derive each of the Keck and CFHT surface density profiles. This approach suppresses fluctuations in the surface brightness profile caused by the small number of red giants in this low-luminosity object.

How does our surface brightness profile compare with previous findings? Unfortunately, Ortolani et al. (1985) and Siegel et al. (2001) each presented surface density profiles 


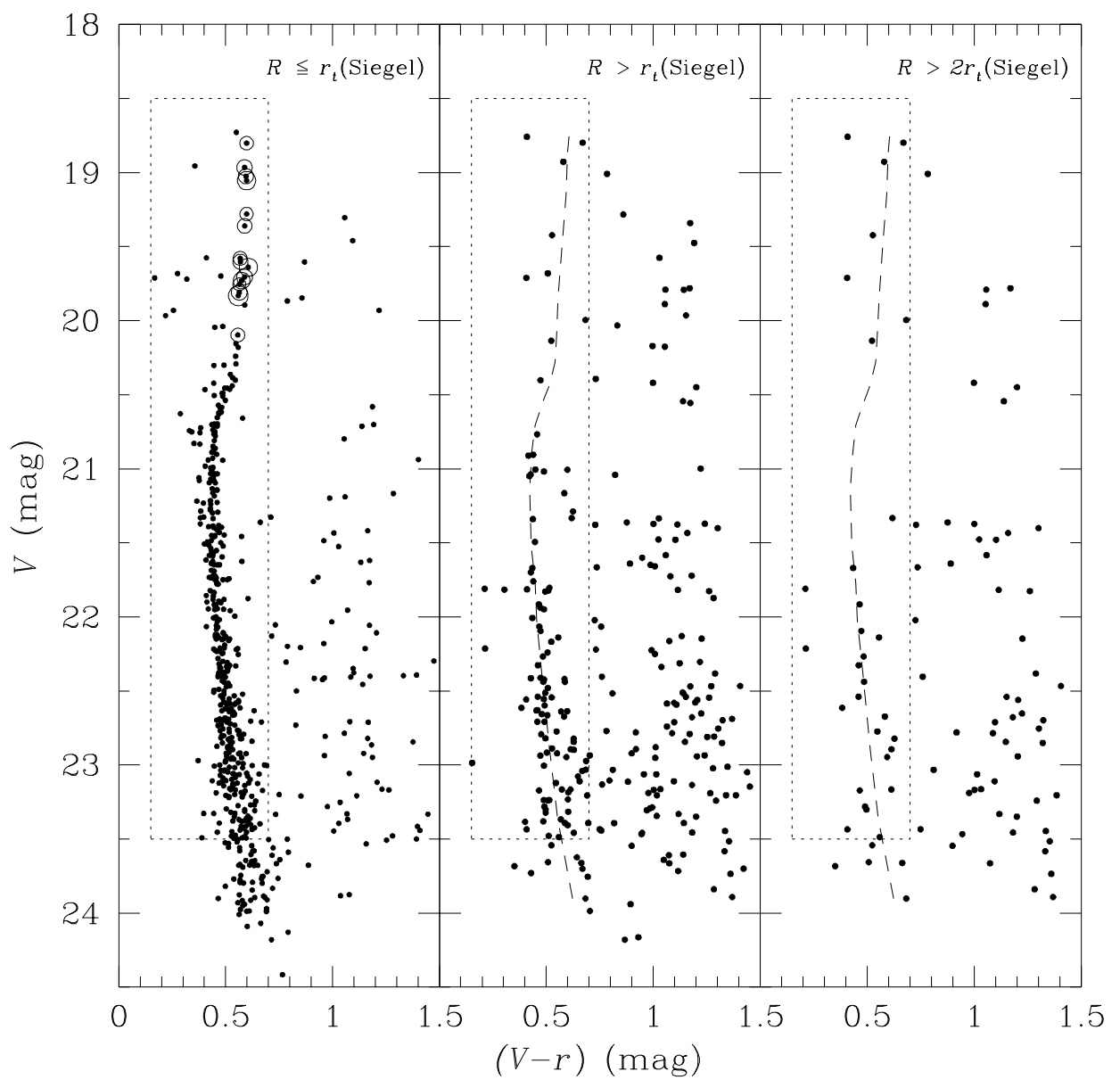

FIG. 6.- Instrumental $V,(V-r)$ color-magnitude diagrams based on our CFHT images in three radial regimes. The left panel shows all objects within the tidal radius, $r_{t}=188^{\prime \prime}$, reported by Siegel et al. (2001). The dotted lines shows the magnitude and color limits used to reject probable non-members. Circled points indicate probable radial velocity members for which we have reliable CFHT photometry. All objects located beyond this radius are plotted in the middle panel, where the dashed curve shows the Palomar 13 fiducial sequence from the previous panel. Objects at distances more than twice the tidal radius of Siegel et al. (2001) are plotted in the right panel.

for Palomar 13, but neither reported surface brightness measurements. Webbink (1985) quotes a central surface brightness of $\mu_{V}=22.41 \mathrm{mag} \operatorname{arcsec}^{-2}$ based on the star counts of Ortolani et al. (1985) and the aperture photometry of Racine (1975). Trager et al. (1995) found a similar core radius and concentration (i.e., $r_{c}=29^{\prime \prime}$ and $c=0.66$ ) but a much different central surface brightness: $\mu_{V}=24.31 \mathrm{mag}$ $\operatorname{arcsec}^{-2}$. The overall shape of the Trager et al. (1995) surface brightness profile, shown by the open stars in Figure 7, is in reasonable agreement with the profile found here but differs systematically by a factor of $\sim 3.4$ in surface brightness (in the sense that their measurements are lower). Their profile consists primarily of star counts (Kinman \& Rosino 1962), normalized to the aperture photometry of Racine (1975). We believe that the normalization given here is to be preferred since two independent measurements (from Keck and CFHT) are in close agreement. Moreover, an integration of the best-fit King-Michie model of Trager et al. (1995) yields an absolute magnitude for Palomar 13 of $M_{V}=-1.25 \mathrm{mag}$. This is $\sim 10$ times fainter than the value of $M_{V}=-3.6$ reported by Ortolani et al. (1985) and the value of $M_{V}=-3.8$ found here, both of which were obtained by direct integration of the luminosity function.

The various curves shown in Figure 7 represent different models for the light distribution in Palomar 13. We will discuss these models in detail below (in $\S 4-5$ ), but before doing so, we shall use the radial velocities presented in Table 2 to investigate the internal kinematics of Palomar 13.

\subsection{Internal Kinematics}

Using our sample of 21 members (V2 excluded), we calculate a systemic velocity of $\left\langle v_{r}\right\rangle_{s}=24.1 \pm 0.5 \mathrm{~km} \mathrm{~s}^{-1}$ using the maximum likelihood estimator of Pryor \& Meylan (1993). The corresponding estimate for the projected intrinsic velocity dispersion using this same sample is $\sigma_{p}=2.2 \pm 0.4 \mathrm{~km} \mathrm{~s}^{-1}$. Omitting ORS-118 from the sample (see $\S 3.1$ ) has essentially no effect on the derived parameters: $\left\langle v_{r}\right\rangle=24.0 \pm 0.5 \mathrm{~km} \mathrm{~s}^{-1}$ and $\sigma_{p}=2.3 \pm 0.4 \mathrm{~km} \mathrm{~s}^{-1}$. Likewise, if ORS-32 is omitted from the sample, the best-fit parameters do not change significantly: $\left\langle v_{r}\right\rangle=24.3 \pm 0.5$ $\mathrm{km} \mathrm{s}^{-1}$ and $\sigma_{p}=2.0 \pm 0.4 \mathrm{~km} \mathrm{~s}^{-1}$.

In the upper panel of Figure 8, we show the radial velocities for these stars plotted against distance from the center of Palomar 13. The horizontal dashed line shows the systemic velocity, $\left\langle v_{r}\right\rangle_{s}=24.1 \mathrm{~km} \mathrm{~s}^{-1}$, while the vertical arrows at $R=1^{\prime}$ denote the radius where the possible excess in the surface density profile begins. It is perhaps noteworthy that the stars with the largest velocity residuals are located at, or beyond, this radius. We note that 


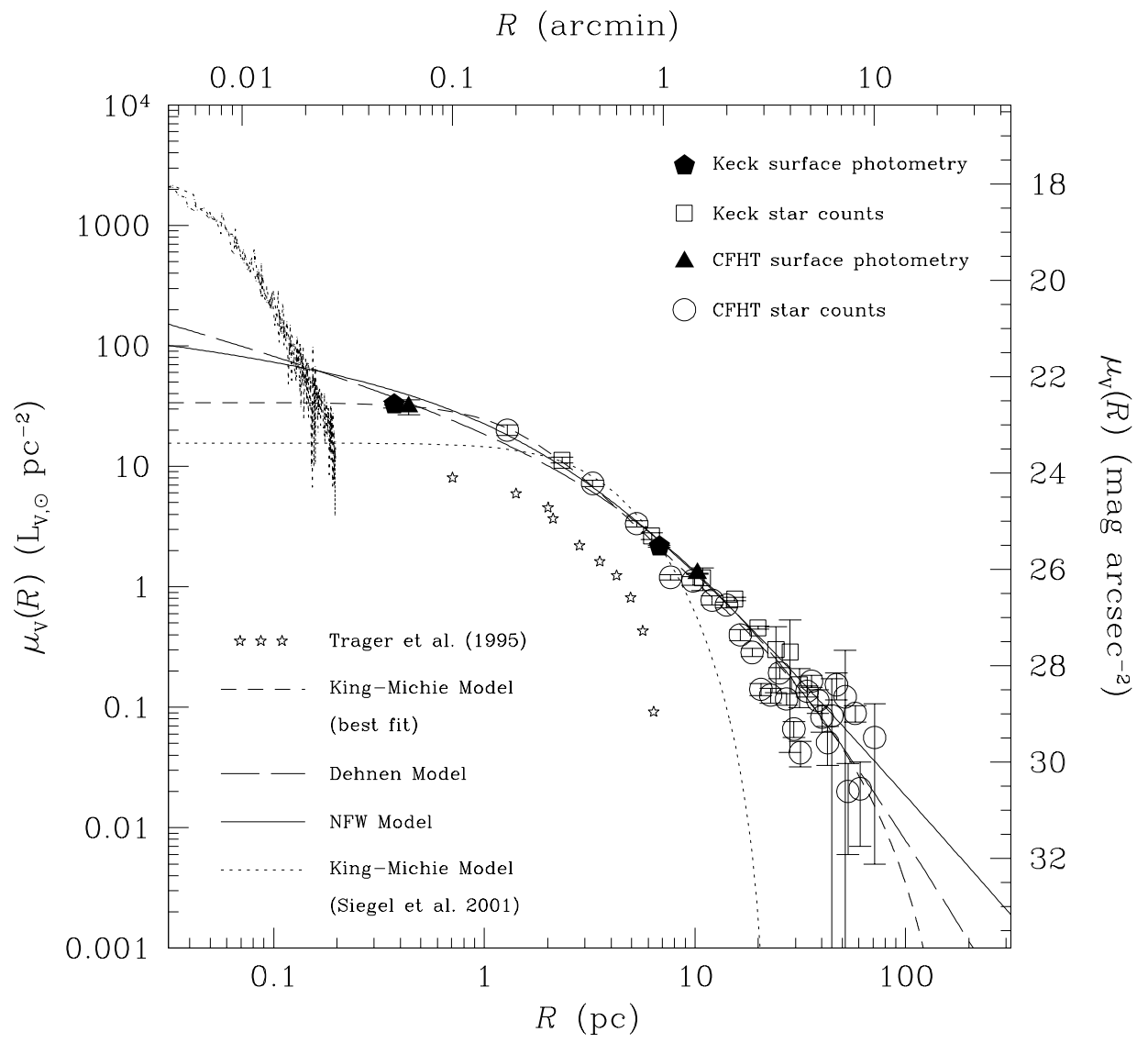

Fig. 7.- $V$-band surface brightness profile for Palomar 13. Pentagons and squares represent surface photometry and star counts based on our Keck imaging, respectively. Surface photometry and star counts using our CFHT imaging are indicated by the triangles and circles. The King-Michie model that best fits this surface brightness profile is shown by the dashed curve. By contrast, the King-Michie model with structural parameters found by Siegel et al. (2001) from their surface density profile (see Fig. 5) is indicated by dotted curve, shifted in the vertical direction. The solid curve shows the NFW model that best fits the surface density profile, while the long-dashed curve shows the best-fit Dehnen model. The dotted curves at the cluster center shows typical stellar profiles on our Keck and CFHT images, scaled to the luminosity of V2, the bright variable that defines the cluster center.

there is a tendency for stars with the most discrepant velocities to also have the smallest uncertainties; this is a consequence of the fact that these stars were observed on multiple observing runs to confirm the first-epoch velocities and to search for any evidence of binarity. As with the full sample, none of these stars shows compelling evidence for radial velocity variations.

In the lower panel of Figure 8, we plot the velocity dispersion profile for Palomar 13. The open circle denotes the dispersion found using our complete sample of 21 members, plotted at their mean radius: $\langle R\rangle=0$ '.84 $(5.9 \mathrm{pc})$. The filled circles show the results of dividing the sample into two radial bins containing 11 and 10 stars, respectively. In this case, the dispersions are $1.0 \pm 0.4 \mathrm{~km} \mathrm{~s}^{-1}$ at $\langle R\rangle=0.36(2.6$ pc) and $2.6 \pm 0.6 \mathrm{~km} \mathrm{~s}^{-1}$ at $\langle R\rangle=1.36(9.6 \mathrm{pc})$. Although there may be some evidence for a rising velocity dispersion profile, we caution that this result is based on a small sample and that the uncertainties in the measured dispersions are appreciable.

How does the measured velocity dispersion for Palomar 13 compare with expectations? Globular clusters are well described by King-Michie models, in which the central velocity dispersion (in kilometers per second) may be approximated by

$$
\sigma_{p, 0}^{2} \simeq 0.003 \Upsilon_{V} r_{c} \mu_{V, 0},
$$

where $\Upsilon_{V}$ is the $V$-band mass-to-light ratio, $r_{c}$ is the core radius in $\mathrm{pc}$, and $\mu_{V, 0}$ is the central surface brightness is units of $L_{V, \odot} \mathrm{pc}^{-2}$ (e.g., Richstone \& Tremaine 1986; Mateo et al. 1991). Based on the King-Michie model fits to the observed surface brightness profile presented in $\S 3.4$ and Table 3, the expected velocity dispersion of Palomar 13 is $\sigma_{p, 0} \simeq 0.6 \mathrm{~km} \mathrm{~s}^{-1}$ for an assumed mass-to-light ratio of $\Upsilon_{V}=2$. Thus, our measured velocity dispersion exceeds the expected value by roughly a factor of 4 . The implications of this finding are presented below, along with a more detailed analysis of the internal kinematics Palomar 13.

\section{MASS-TO-LIGHT RATIO}

As discussed in $\S 3.4$, our new surface density profile for Palomar 13 suggests that either a significant fraction of its stars are located beyond the ostensible tidal radius, or the object is more spatially extended than previously believed. We now examine its internal kinematics within the context of these two scenarios.

The short-dashed curve in Figure 7 shows the KingMichie model that best fits the observed surface brightness profile for Palomar 13. The parameters for this model are $r_{c}=14^{\prime \prime} \pm 2^{\prime \prime}, c=2.0 \pm 0.15$, and $\mu_{V, 0}=22.54_{-0.17}^{+0.30} \mathrm{mag}$ $\operatorname{arcsec}^{-2}$. (These and other model parameters are summarized in Table 3, along with various observed and derived 


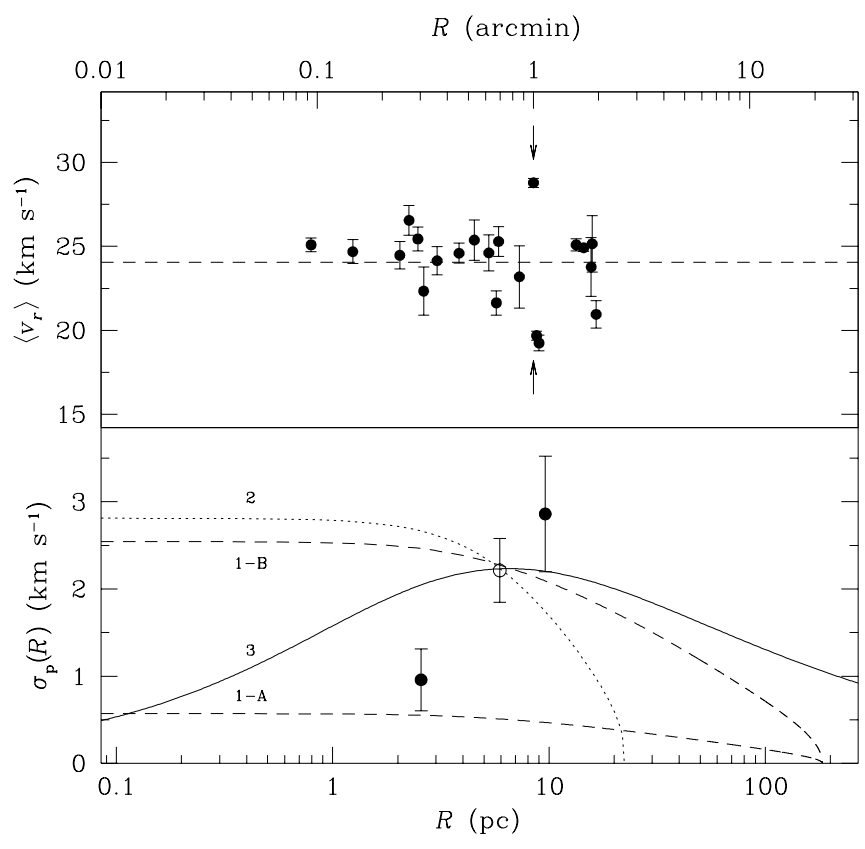

FIG. 8.-Top: Heliocentric radial velocity for probable cluster members plotted against distance from the cluster center (filled circles). The dashed line indicated the cluster's systemic velocity. The arrows denote the approximate "break" radius, $R_{b} \simeq 1^{\prime}$, where the possible excess in the cluster's surface density profile begins. Bottom: Velocity dispersion profile for Palomar 13. The open circle shows the intrinsic velocity dispersion for the entire sample of stars, plotted at the mean radius of the sample. The two filled circles show the intrinsic velocity dispersion that is found if the sample is divided into two radial bins containing 11 and 10 stars each. The different curves (1-A, 1-B, 2, and 3) indicate various model fits to the data; a detailed description of each model is presented in the text.

properties of Palomar 13.) According to this model, Palomar 13 has both a higher concentration and a greater spatial extent $\left(r_{t} \sim 180 \mathrm{pc}\right)$ than suggested by previous studies. Indeed, this concentration index is considerably larger than that expected for a globular cluster of this luminosity or surface brightness (see, e.g., Djorgovski 1991).

Integration of this profile yields a total luminosity of $L_{V} \simeq(2.8 \pm 0.4) \times 10^{3} L_{V, \odot}$. The corresponding velocity dispersion profile for an assumed mass-to-light ratio of $\Upsilon_{V}=2$ is shown by the lower dashed curve in the bottom panel of Figure 8 (curve 1-A). In agreement with the naive analysis presented above, we find the measured velocity dispersion to exceed that expected for a normal globular cluster of this concentration and luminosity. A maximum likelihood solution for the best-fit scale velocity of this same King-Michie model gives the upper dashed curve shown in this figure (curve 1-B). This model has a central velocity dispersion of $\sigma_{p, 0} \simeq 2.5 \mathrm{~km} \mathrm{~s}^{-1}$ and a total mass of $M=\left(1.10_{-0.36}^{+0.44}\right) \times 10^{5} M_{\odot}$. The mass-to-light ratio is then $\Upsilon_{V}=40_{-17}^{+2 \overline{4}}(1 \sigma$ uncertainties $)$.

Alternatively, we may use the King-Michie model suggested by Siegel et al. (2001) to estimate the mass and massto-light ratio of Palomar 13. This model, which is shown by the dotted curve in Figure 7, yields a total luminosity of $L_{V} \simeq 1.2 \times 10^{3} L_{V, \odot}$. A maximum likelihood scaling of the model velocity dispersion profile to the measured velocities produces the dotted curve in the lower panel of Figure 8 (curve 2). For this model, which has a central velocity dispersion of $\sigma_{p, 0} \simeq 2.8 \mathrm{~km} \mathrm{~s}^{-1}$, the mass within the tidal radius $\left(r_{t}=188^{\prime \prime}\right.$ or $\left.23 \mathrm{pc}\right)$ is $M=\left(5.5_{-1.6}^{+1.9}\right) \times 10^{4} M_{\odot}$. The corre- sponding mass-to-light ratio is $\Upsilon_{V}=48_{-14}^{+17}$ where the quoted $1 \sigma$ uncertainties refer only to the errors on the derived mass. Thus, irrespective of the adopted surface brightness profile, it is not possible to reproduce the observed velocity dispersion with a mass-to-light ratio that is typical of globular clusters (e.g., $\left\langle\Upsilon_{V}\right\rangle=2.3 \pm 1.1$; Pryor \& Meylan 1993).

\section{IMPLICATIONS}

What are the implications of this finding? We begin by examining - and tentatively rejecting - the possibility that the observed velocity dispersion has been inflated by a "velocity jitter" in the atmospheres of the program stars or by a population of spectroscopic binary stars. We then examine other scenarios that may explain the observed properties of Palomar 13, including tidal disruption, a mass function that is heavily skewed to low-mass stars or heavy stellar remnants, modified Newtonian dynamics, and/or the presence of a massive dark halo. It is worth bearing in mind that these various possibilities may not be mutually exclusive (i.e., a high binary fraction combined with tidal heating and mass loss).

\subsection{Velocity Jitter and/or Binary Stars?}

The value of $\sigma_{p}=2.2 \pm 0.4 \mathrm{~km} \mathrm{~s}^{-1}$ reported in $\S 3.5$ refers to the intrinsic velocity dispersion for Palomar 13; i.e., the contribution from measurement uncertainties has been removed (see Pryor \& Meylan 1993). Our velocity uncertainties have been calculated empirically from 53 distinct radial velocities of faint metal-poor stars (accumulated during multiple observing runs), so we are confident that the measured dispersion is not the result of underestimated velocity uncertainties. Such an explanation would require $\alpha \simeq 20 \mathrm{~km} \mathrm{~s}^{-1}$ (see eq. [2]), whereas we measure $\alpha=9.0 \mathrm{~km}$ $\mathrm{s}^{-1}$ and find $\alpha \leq 12.8 \mathrm{~km} \mathrm{~s}^{-1}$ at $99 \%$ confidence.

Bright red giants in globular clusters often show evidence for radial velocity variations in the range $2-5 \mathrm{~km} \mathrm{~s}^{-1}$ (Gunn \& Griffin 1979; Mayor et al. 1984). This so-called velocity jitter is thought to arise from pulsational motions in the atmospheres of these evolved stars. It is unlikely that our measured velocity dispersion is the result of such velocity variations since long-term monitoring of red giants in nearby globular clusters has established that the magnitude of this jitter shows a strong luminosity dependence; i.e., it is significant at the $1 \mathrm{~km} \mathrm{~s}^{-1}$ level only for stars that are located within $\sim 1$ mag of the RGB tip. Our radial velocity sample includes no stars this bright. In fact, our most luminous member (ORS-118) has $M_{V} \simeq-0.3 \mathrm{mag}$, a full $2.3 \mathrm{mag}$ below the tip of the RGB at this metallicity.

Might our estimate for the velocity dispersion be inflated by "dynamically hard" spectroscopic binary stars residing in the cluster? There are reasons to believe that the binary star fraction in Palomar 13 may be appreciable. First, the CMD of Palomar 13 shows both a sizeable blue straggler population and a possible "second sequence"-features that are usually interpreted as signatures of unresolved binaries (Mateo et al. 1990; Romani \& Weinberg 1991). Second, on purely theoretical grounds, the low stellar density and velocity dispersion of Palomar 13 (for an assumed "normal" mass-to-light ratio) suggest that even wide binaries might have survived disruption over a Hubble time. For instance, for a central velocity dispersion of $\sigma_{p, 0}=$ 
TABLE 3

Observed and Derived Parameters for Palomar 13

\begin{tabular}{|c|c|c|}
\hline Parameter & Value & Reference \\
\hline Right ascension $\alpha(\mathrm{J} 2000)$.. & $23^{\mathrm{h}} 06^{\mathrm{m}} 44^{\mathrm{s}} .8$ & 1 \\
\hline Declination $\delta(J 2000)$ & $+12^{\circ} 46^{\prime} 18^{\prime \prime}$ & 1 \\
\hline Apparent distance modulus $(m-M)_{V}(\mathrm{mag})$ & $17.27 \pm 0.1$ & 2 \\
\hline 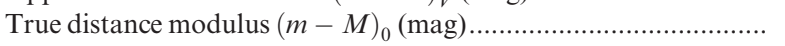 & $16.93 \pm 0.1$ & 2 \\
\hline 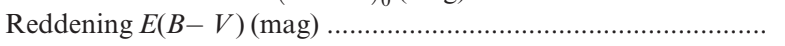 & $0.11 \pm 0.02$ & 3 \\
\hline 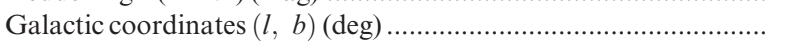 & $(87.1,-42.7)$ & 1 \\
\hline 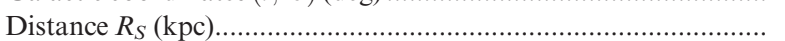 & $24.3_{-1.1}^{+1.2}$ & 2 \\
\hline Galactocentric distance $R_{G}(\mathrm{kpc})$. & $25.3_{-1.1}^{+1.2}$ & 2 \\
\hline Metallicity $[\mathrm{Fe} / \mathrm{H}](\mathrm{dex})$ & $-1.9 \pm 0.2$ & 2 \\
\hline 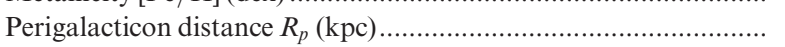 & 11.2 & 1 \\
\hline 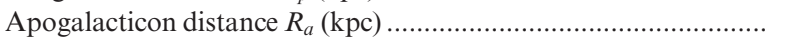 & 80.2 & 1 \\
\hline Orbital period $P(\mathrm{Gyr})$ & 1.1 & 1 \\
\hline 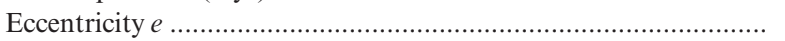 & 0.76 & 1 \\
\hline 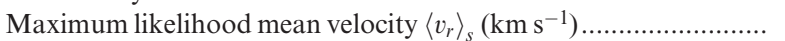 & $24.1 \pm 0.5$ & 2 \\
\hline 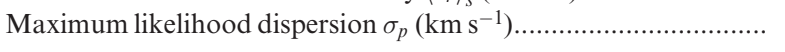 & $2.2 \pm 0.4$ & 2 \\
\hline \multicolumn{3}{|l|}{ Siegel et al. (2001) King-Michie Model } \\
\hline Core radius $r_{c}(\operatorname{arcsec}) .$. & 39 & 1 \\
\hline Core radius $r_{c}(\mathrm{pc}) \ldots \ldots \ldots$ & 4.6 & 1 \\
\hline Tidal radius $r_{t}(\operatorname{arcsec}) \ldots$ & 188 & 1 \\
\hline Tidal radius $r_{t}(\mathrm{pc}) \ldots \ldots$. & 23 & 1 \\
\hline Central projected luminosity density $\mu_{V, 0}\left(\operatorname{mag} \operatorname{arcsec}^{-2}\right) \ldots \ldots \ldots \ldots \ldots$ & 23.39 & 2 \\
\hline Central projected luminosity density $\mu_{V, 0}\left(L_{V, \odot} \mathrm{pc}^{-2}\right) \ldots \ldots \ldots \ldots \ldots \ldots \ldots$ & 15 & 2 \\
\hline Concentration $c$ & 0.7 & 1 \\
\hline 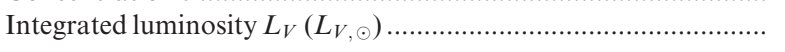 & $1.2 \times 10^{3}$ & 1 \\
\hline \multicolumn{3}{|l|}{ Best-fit King-Michie Model } \\
\hline Core radius $r_{c}(\operatorname{arcsec}) \ldots$ & $14 \pm 2$ & 2 \\
\hline Core radius $r_{c}(\mathrm{pc}) \ldots \ldots \ldots \ldots \ldots \ldots \ldots \ldots \ldots$ & $1.70 \pm 0.24$ & 2 \\
\hline Tidal radius $r_{t}(\operatorname{arcmin}) \ldots . . . .$. & $26 \pm 6$ & 2 \\
\hline Tidal radius $r_{t}(\mathrm{pc})$ & $182 \pm 41$ & 2 \\
\hline 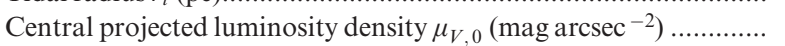 & $22.54_{-0.17}^{+0.20}$ & 2 \\
\hline Central projected luminosity density $\mu_{V, 0}\left(L_{V, \odot} \mathrm{pc}^{-2}\right) \ldots \ldots \ldots \ldots \ldots \ldots$ & $33.8 \pm 5.8$ & 2 \\
\hline 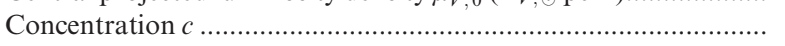 & $2.0 \pm 0.15$ & 2 \\
\hline 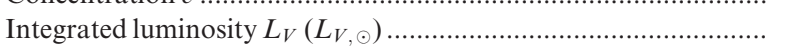 & $(2.8 \pm 0.4) \times 10^{3}$ & 2 \\
\hline 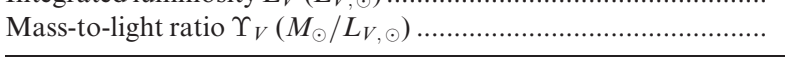 & $40_{-17}^{+24}$ & 2 \\
\hline \multicolumn{3}{|l|}{ Dehnen Model } \\
\hline 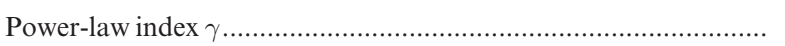 & $1.46 \pm 0.06$ & 2 \\
\hline Scale radius $a(\operatorname{arcsec})$ & $135 \pm 9$ & 2 \\
\hline Scale radius $a(\mathrm{pc})$ & $15.9 \pm 1.0$ & 2 \\
\hline 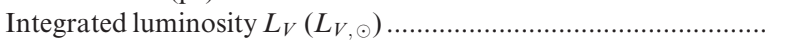 & $(3.5 \pm 0.1) \times 10^{3}$ & 2 \\
\hline \multicolumn{3}{|l|}{ NFW Model } \\
\hline Scale radius $r_{s}(\operatorname{arcsec}) \ldots \ldots \ldots \ldots$ & $24 \pm 1$ & 2 \\
\hline Scale radius $r_{s}(\mathrm{pc})$ & $2.8 \pm 0.1$ & 2 \\
\hline Central luminosity density $\rho_{V}^{0}\left(L_{V, \odot} \mathrm{pc}^{-3}\right) \ldots$ & $4.3 \pm 0.4$ & 2 \\
\hline 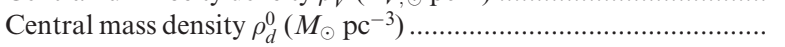 & $80 \pm 31$ & 2 \\
\hline Central mass-to-light ratio $\Upsilon_{V}\left(M_{\odot} / L_{V, \odot}\right) \ldots \ldots \ldots$ & $19_{-7}^{+8}$ & 2 \\
\hline
\end{tabular}

REFERENCES.-(1) Siegel et al. 2001; (2) This paper; (3) Schlegel et al. 1998.

$1 \mathrm{~km} \mathrm{~s}^{-1}$, a pair of equal-mass $0.8 M_{\odot}$ stars would be disrupted by encounters with other members only if separated by more than $a_{\text {crit }} \sim 1400 \mathrm{AU}$ (Hills 1984). Such binaries, if on circular orbits, would have periods of $P \sim 4 \times 10^{4} \mathrm{yr}$ and orbital velocities of $v \sim 1 \mathrm{~km} \mathrm{~s}^{-1}$, i.e., modest, but potentially important, given the low dispersion expected for Palomar 13.

To explore the possible effects of binary stars on our measured velocity dispersion, we have carried out Monte Carlo simulations similar to those described in Pryor, Latham, \&
Hazen (1988) and Côté et al. (1996). In brief, we have generated simulated radial velocity measurements having the identical precision and temporal spacing as the actual data set. For each simulation, we take the intrinsic velocity dispersion to that predicted by a King-Model having $\Upsilon_{V} \simeq 2$ and calculate the contribution to the measured velocity dispersion of a randomly drawn percentage, $f_{b}$, of unresolved spectroscopic binary stars. Binaries are assumed to have circular orbits. The orbital periods in years, $P$, and mass ratios, $q=M_{2} / M_{1}$, are drawn at random over the ranges 
$-1.0 \leq \log P \leq 4.0$ and $-0.6 \leq \log q \leq 0.0$. For each $f_{b}$, we generate 1000 artificial data sets and calculate the mean cluster dispersion in precisely the same manner as the actual for the actual data; the percentage of binaries is varied until the median dispersion found in 1000 simulated data sets is equal to the value obtained using the actual observations. These simulation suggest that a value of $f_{b} \simeq 30 \%$ produces a median velocity dispersion that best matches the observed value of $\sigma_{p}=2.2 \mathrm{~km} \mathrm{~s}^{-1}$. If it is assumed that the binaries do not have circular orbits but instead have a "thermal" distribution of eccentricities, then the percentage of binaries needed to explain the measured dispersion would be roughly twice as large (see Pryor et al. 1988; Côté et al. 1996).

By definition, the velocity dispersion profile predicted by these simulations differs from that of the input King-Michie model only in normalization, since we have made the firstorder assumption that the binaries have the same density distribution as the single stars. However, if the binaries are instead assumed to be more centrally concentrated than the other stars - perhaps as a result of mass segregation - then the expected velocity dispersion profile would show a steeper decline. Needless to say, a rising velocity dispersion profile would prove difficult to reconcile with this scenario. We find weak evidence for an outward increase in the observed velocity dispersion of Palomar 13, but given the small number of measured velocities, this feature clearly needs to be confirmed by additional observations. As Figure 6 demonstrates, there are many promising candidates beyond $R \sim 2^{\prime}$ (i.e., the radii of the most distant members in the current radial velocity sample). It would be of considerable interest to measure velocities for these stars in order to determine if they are members of Palomar 13 and, if so, to see if the velocity dispersion profile rises with increasing radius.

Likewise, it would be desirable to search directly for velocity variations associated with possible orbital motions of these stars. Unfortunately, the long periods of the putative binaries would render any such search hopeless; a more promising approach would be to measure $f_{b}$ by modeling the "second sequence" measured from high-resolution Hubble Space Telescope (HST) imaging (e.g., Rubenstein \& Bailyn 1997).

Finally, we note that spectroscopic binaries may offer a viable explanation for the observed high-velocity dispersion, but they cannot on their own explain the anomalous surface density profile.

\subsection{Disruption?}

An underlying assumption of the dynamical analysis presented in $\S 4$ is that of virial equilibrium. In light of the large velocity dispersion measured for Palomar 13, it is prudent to investigate the validity of this assumption, which has recently been called into question for many of the dwarf satellites and globular clusters in the Galactic halo (Majewski et al. 2001). Simply put, could Palomar 13 be in the process of disruption? This possibility was advocated by Siegel et al. (2001), who combined their proper motion measurements with the systemic radial velocity presented here to derive an orbit for Palomar 13. They concluded that Palomar 13 is just $\sim 70 \mathrm{Myr}$ past perigalacticon $\left(R_{p} \simeq 11.2 \mathrm{kpc}\right)$ and suggested that it has undergone catastrophic tidal heating during its recent approach. Since $N$-body simulations of tidal tails in globular clusters (Combes, Leon, \& Meylan 1999) show that the time taken for evaporating stars to diffuse along the cluster orbit can exceed this timescale, it is worth examining this possibility further.

Siegel et al. (2001) noted the presence of a population of "extra-tidal" stars in their surface density profile. We too find a possible excess in the Palomar 13 surface density profile, beginning at $R \sim 1^{\prime}$. This feature is evident in both the CFHT and LRIS surface density profiles, and the CFHT photometry suggests that many of these stars have magnitudes and colors that are consistent with those expected for Palomar 13 members (see $\S 3.4$ ). Thus, we confirm the existence of this feature; the issue is whether the stars associated with it are truly evaporating from Palomar 13, or if they are instead bound members.

As shown in Figure 5, the surface density profile for Palomar 13 shows a roughly power-law decline for $R \gtrsim 0 ! 3$. This behavior is most pronounced beyond $R \sim 1^{\prime}$, where there may be some evidence for a "break" in the profile. Johnston, Sigurdsson, \& Hernquist (1999) and Johnston, Choi, $\&$ Guhathakurta (2001) have carried out $N$-body simulations of satellites orbiting in the Galactic tidal field; they find that the radial distribution of unbound stars in the outer regions of disrupting satellites should define a power-law profile - broadly similar to what we observe for Palomar 13. The dashed lines in Figure 5 show power-law profiles, $\Sigma(R) \propto R^{-\gamma}$, having representative exponents of $\gamma=0.5$, 1.5 , and 2.5. Over the range $1 \lesssim R \lesssim 4$, we measure $\gamma=$ $1.8 \pm 0.2$ for Palomar 13. This value is steeper than the formal value of $\gamma \simeq 1$ estimated by Johnston et al. (1999) but is probably consistent with expectations given uncertainties in the numerical treatment (e.g., the initial density profile, viewing geometry, and orbital phase; Johnston et al. 2001).

If this interpretation is correct, then a crude estimate for the mass loss rate may be made as follows. Johnston et al. (1999) present two simple expressions for the fractional mass loss rate using extra-tidal stars,

$$
\begin{aligned}
& (d f / d t)_{1}=\cos (\theta) \pi R_{b} N_{\mathrm{xt}} /\left[\left(R_{\mathrm{xt}}-R_{b}\right) N_{b} P\right], \\
& (d f / d t)_{2}=\cos (\theta) \Sigma\left(R_{b}\right) 2 \pi R_{b}^{2} /\left[N_{b} P\right],
\end{aligned}
$$

where $\theta$ is the angle subtended by our line of sight and the plane perpendicular to the satellite's motion, $R_{b}$ is the "break" radius where the surface density excess begins, $R_{\mathrm{xt}}$ is the radius where the profile of the extra-tidal component ceases to be well defined, $N_{\mathrm{xt}}$ is the number of stars in the range $R_{b} \leq R \leq R_{\mathrm{xt}}$, and $P$ is the satellite's azimuthal period (taken to be 2.0 Gyr following Dinescu et al. 2001). Using the space velocity of Siegel et al. (2001) and the surface density profile shown in Figure 5, we find $(d f / d t)_{1} \sim 0.2 \mathrm{Gyr}^{-1}$ and $(d f / d t)_{2} \sim 0.15 \mathrm{Gyr}^{-1}$. There are significant uncertainties involved in these calculations, but it is clear that if this interpretation of the surface density profile is correct, then both estimators point to enormous recent mass loss. The inferred mass loss rate is far higher than those estimated by Leon, Meylan, \& Combes (2000) from star counts in a sample of 20 Galactic globular clusters; for example, in the case of $\omega$ Centauri, Leon et al. (2000) find that only $\approx 1 \%$ of the cluster's mass has been lost during the last tidal encounter. Taken at face value, the mass loss rates for Palomar 13 suggest that it has suffered severe heating and will disrupt entirely within the next few gigayears.

On the other hand, there may be some difficulties with this interpretation. $N$-body simulations of the tidal heating of satellites indicate that the tidally stripped material tends to 


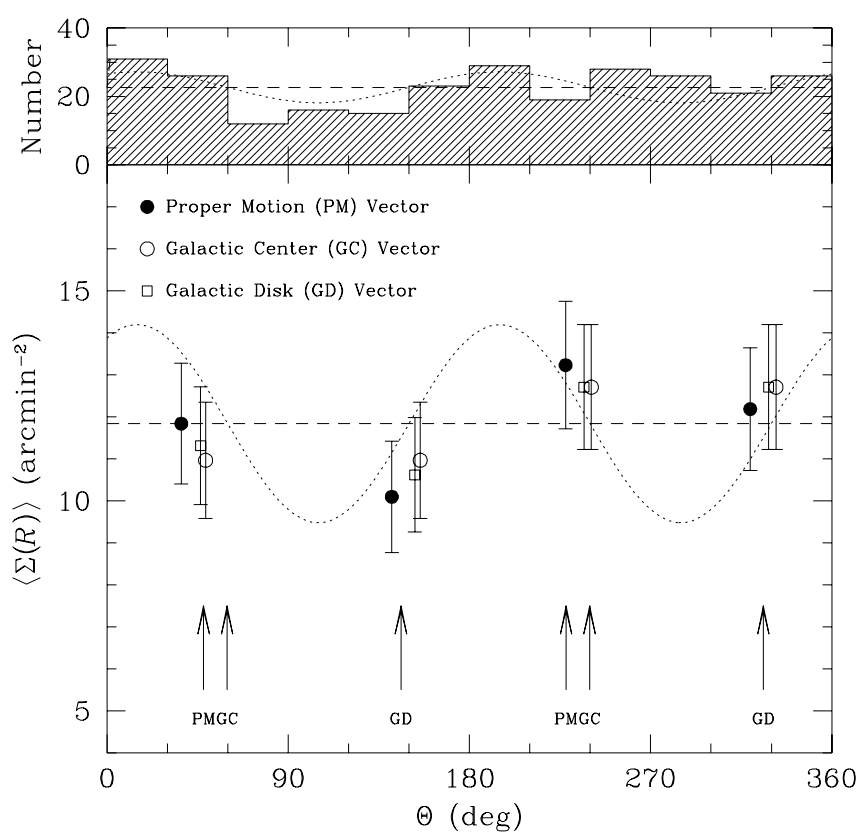

FIG. 9.-Top: Histogram of position angles for stars in our photometric database that are located in the radial range $1^{\prime} \leq R \leq 2$ !' 88 . The dashed line shows the mean number of stars per azimuthal bin, while the dotted curve shows the best-fit sinusoid: $N \propto(4.5 \pm 1.7) \sin \left[2 \Theta+\left(60^{\circ} \pm 11^{\circ}\right)\right]$. This curve has density maxima at $\Theta_{p}=15^{\circ}$ and $195^{\circ}$. Bottom: Mean stellar surface density in the range $1^{\prime}<R<2$ ! 88 for each of four $90^{\circ}$ sectors centered on cluster center. The dashed and dotted curves correspond to the mean surface density and sinusoid described in the upper panel (i.e., with a bestfit amplitude of $2.4 \pm 0.9 \mathrm{arcmin}^{-2}$ ). Surface densities are shown for three different orientations of the quadrants: in the first case (filled circles), the sector boundaries are offset by $\pm 45^{\circ}$ from the position angle of the vector that points in the direction of Palomar 13's absolute proper motion (i.e., Fig. 1, short arrow). In the second case (open circles), the boundaries are offset by $\pm 45^{\circ}$ from the position angle of the vector which points in the direction of the Galactic center (i.e., Fig. 1, long arrow). In the final case (open squares), the boundaries are offset by $\pm 45^{\circ}$ from the position angle of the vector which points in the direction perpendicular to the Galactic disk (i.e., Fig. 1, intermediate arrow). The orientations of these three vectors are indicated by the respective arrows. There is no compelling evidence for a preferred orientation of Palomar 13 stars in this radial range.

diffuse along the orbits, forming leading and trailing streams. We have examined our star counts for evidence of such streams by measuring the mean surface density of stars in four quadrants over the radial range $1^{\prime} \leq R \leq 2$ ! 88 . The inner limit corresponds to the break radius, $R_{b}$, while the outer limit is the most distant radius at which our geometric coverage is still $100 \%$ complete. The results of this exercise are shown in Figure 9. We find only weak evidence that these stars are oriented along a preferred direction. The density maxima of the best-fit sinusoid shown in this figure are found at position angles of $\Theta_{p}=15^{\circ} \pm 11^{\circ}$ and $195^{\circ} \pm 11^{\circ}$. The agreement between this orientation and those of the absolute proper motion vector, $\Theta_{p}=48^{\circ}$, and the Galactic center direction, $\Theta_{p}=240^{\circ}$, is unexceptional; i.e., the differences in position angles are $\Delta \Theta_{p}=33^{\circ} \pm 11^{\circ}$ and $45^{\circ} \pm 11^{\circ}$, respectively. There is also poor agreement with the vector perpendicular to the Galactic disk, which has $\Theta_{p}=327^{\circ}$. If the peculiar surface density profile of Palomar 13 is indeed the result of tidal heating, then this lack of a preferred orientation for the putative extra-tidal stars is somewhat puzzling. It would be worthwhile investigating this issue using star counts that extend over a wider field, preferably well

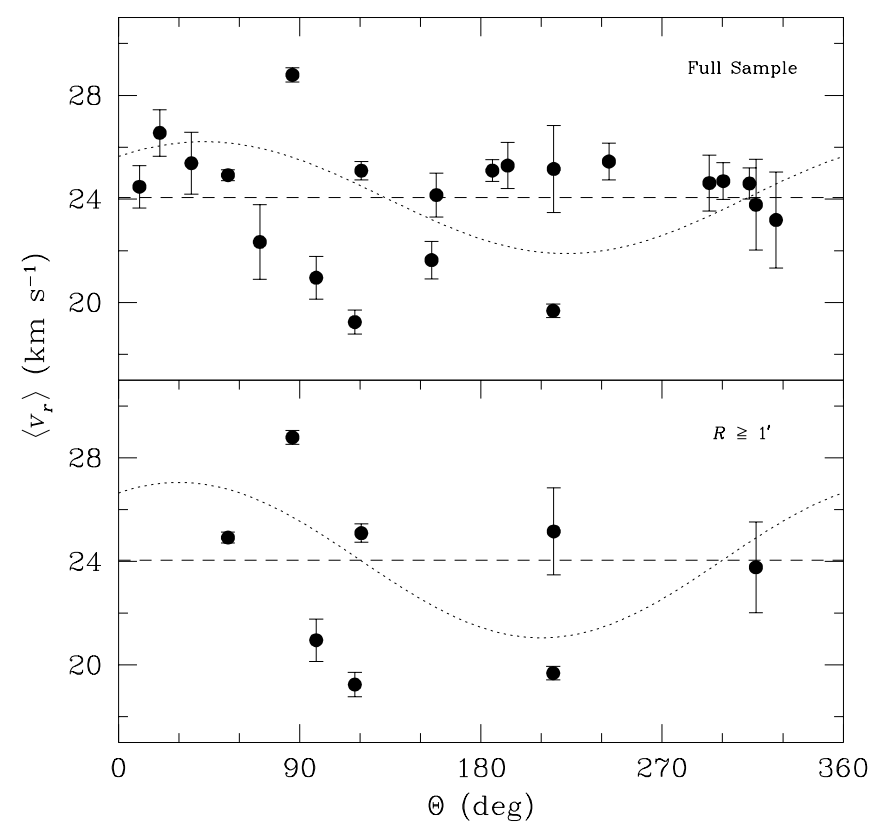

FIG. 10.-Top: Mean radial velocity vs. position angle, $\Theta$, for the sample of 21 probable member stars. The dashed line indicates the systemic velocity, while the dotted curve shows the best-fit sinusoid of the form $\left\langle v_{r}\right\rangle=\left\langle v_{r}\right\rangle_{s}+A \sin \left(\Theta+\Theta_{p}\right)$, where $\left\langle v_{r}\right\rangle_{s} \equiv 24.1 \mathrm{~km} \mathrm{~s}^{-1}$. A weighted leastsquares fit yields $A=2.1 \pm 1.0 \mathrm{~km} \mathrm{~s}^{-1}$ and $\Theta_{p}=48^{\circ} \pm 47^{\circ}$, while an unweighted fit gives $A=0.8 \pm 1.0 \mathrm{~km} \mathrm{~s}^{-1}$ and $\Theta_{p}=104^{\circ} \pm 130^{\circ}$. We conclude that there is no evidence for rotation among the full sample of Palomar 13 members. Bottom: Same as above, except for the eight stars located at, or beyond, the "break radius" of $R_{b} \sim 1$ '. The weighted best-fit sinusoid is shown by the dotted curve, which has parameters $A=3.0 \pm 3.0$ $\mathrm{km} \mathrm{s}^{-1}$ and $\Theta_{p}=60^{\circ} \pm 65^{\circ}$. An unweighted fit gives $A=2.0 \pm 1.7 \mathrm{~km} \mathrm{~s}^{-1}$ and $\Theta_{p}=84^{\circ} \pm 44^{\circ}$. Thus, as with the full sample, we find no evidence for statistically significant rotation for those stars at large radii.

beyond the modest range radial range $(R \lesssim 3)$ examined above.

Piatek \& Pryor (1995) have used $N$-body simulations to show that it is possible to heat the outer regions of satellites during close passages with the Galaxy. Such encounters often have the effect of inducing ordered motions at large radii while leaving the central velocity dispersion unchanged. According to the simulations, the gradient in velocity is usually maximized along the vector that points to the Galactic center. This vector is shown by the long arrow in Figure 1; for comparison, the short arrow shows the direction of the absolute cluster motion as measured by Siegel et al. (2001), while the intermediate arrow denotes the direction perpendicular to the Galactic plane. As shown in Figure 10, we find no statistically significant evidence for "rotation" among our program stars, as might be expected for stars in the leading or trailing streams of a disrupting cluster (see also Fig. 1, where the velocity residuals are illustrated on a star-by-star basis).

Finally, we note that there is an additional (albeit indirect) constraint on the mass and dynamical status of Palomar 13. The tidal radius of a satellite object orbiting in the Galactic potential is given by

$$
r_{t}=R_{p}\left[M / M_{G}(3+e)\right]^{1 / 3},
$$

where $R_{p}$ is the distance from the Galactic center at perigalacticon, $M_{G}$ is the interior Galactic mass at this point, $e$ is the orbital eccentricity of the satellite, and $M$ is its mass 
(King 1962). If we assume a canonical mass-to-light for Palomar 13 (e.g., $\Upsilon_{V}=2$ ), we may use equation (9) to obtain a crude estimate for the expected tidal radius. Adopting $e=0.76$ and $R_{p}=11.2 \mathrm{kpc}$ for Palomar 13 (Siegel et al. 2001), and assuming using $M_{G}\left(R_{p}\right)=\Theta_{c}^{2} R_{p} / G$ (Fich \& Tremaine 1991) and $\Theta_{c}=220 \mathrm{~km} \mathrm{~s}^{-1}$, we find $r_{t} \sim 26 \mathrm{pc}$ for a total luminosity of $L_{V}=3 \times 10^{3} L_{V, \odot}$. This is remarkably close to the tidal radius of $r_{t}=23 \mathrm{pc}$ for the King-Michie model of Siegel et al. (2001), which is shown as the dotted curves in Figures 5 and 7. This is consistent with the view that the excess in the surface density profile is a population of extra-tidal stars. The alternative interpretation-that the much larger tidal radius implied by the best-fit King-Michie model in Figure 7 is evidence for dark matter in Palomar 13 - will be examined in $\S 5.4$

\subsection{Baryonic Dark Matter: Low-Mass Stars and/or Heavy Remnants?}

Taken at face value, the measured velocity dispersion for Palomar 13 implies a total mass of $M \sim$ $\left(1.1_{-0.3}^{+0.4}\right) \times 10^{5} M_{\odot}$, far greater than that expected on the basis of the observed luminosity; i.e., $M \equiv \Upsilon_{V} L_{V} \sim$ $6 \times 10^{3} M_{\odot}$. The obvious baryonic candidates for this additional mass would be low-mass main-sequence stars, or heavy stellar remnants such as white dwarfs and neutron stars. Both varieties of heavy remnants are known to populate globular clusters (see Hut et al. 1992), and the contribution of white dwarfs to the overall mass budget is thought to increase as clusters evaporate (e.g., Vesperini \& Heggie 1997). In this case, the low-luminosity of Palomar 13 might be interpreted as indirect evidence for an advanced stage of dynamical evolution. However, if one accepts the view that Palomar 13 is in dynamical equilibrium (see above), then this would require $\sim 95 \%$ of the total mass to reside in heavy remnants. This percentage is far higher than that found in nearby, well-studied clusters; for example, Meylan (1987, 1989) finds the percentage of heavy remnants (mainly white dwarfs) in 47 Tucanae and $\omega$ Centauri to be $20 \% \pm 5 \%$ and $25 \% \pm 15 \%$, respectively (although the dynamical mass found here for Palomar 13 is only half that contained in heavy remnants in 47 Tuc, by virue of the latter's much larger mass). Unfortunately, a direct search for white dwarfs in Palomar 13 would be extremely challenging since even the brightest such objects are expected to have $V \gtrsim 27$ mag at this distance (Richer et al. 1997). ${ }^{9}$

On the other hand, a determination of the main-sequence luminosity function down to this level (corresponding to $M_{V}=10 \mathrm{mag}$ and $\Upsilon_{V} \simeq 25$; Silvestri et al. 1998) is well within the reach of $H S T$ and could put interesting constraints on the mass contained in low-mass, main-sequence stars. However, as shown in Figure 11, the available data suggest that the main-sequence luminosity function of Palomar 13 is adequately described by a rather flat mass function, with exponent $x \sim 0.0$ over the range $21.5 \lesssim V \lesssim 24$ (equivalent to $0.79 \lesssim M / M_{\odot} \lesssim 0.61$; Bergbusch \& VandenBerg 1992). If the initial mass function of Palomar 13 had a more nearly universal form (for instance, near the "Salpeter value " of $x=1.35$; Salpeter 1955), then the flat mass function we find may be evidence for advanced dynamical evolu-

\footnotetext{
${ }^{9}$ Catalogs of pulsars and X-ray sources associated with globular clusters list no such objects in Palomar 13 (Kulkarni \& Anderson 1996; Verbunt 2001).
}

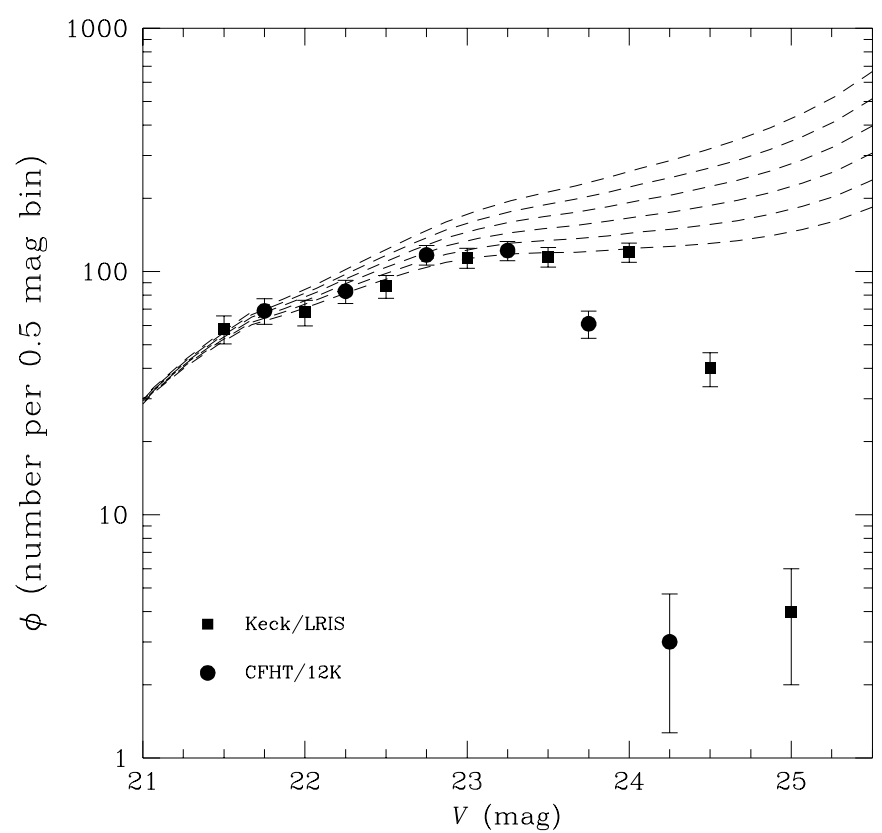

FIG. 11.-Comparison of the observed luminosity function for Palomar 13 with theoretical luminosity functions for $V \gtrsim 21.5 \mathrm{mag}$. Data points from Keck are shown by the squares; circle indicate measurements from CFHT. The theoretical luminosity functions have been generated from the $[\mathrm{Fe} / \mathrm{H}]=-1.78 \mathrm{dex}, 14 \mathrm{Gyr}$ isochrone of Bergbusch \& VandenBerg (1992). Six different values of the mass function exponent, $x$, are shown; from top to bottom, the dashed curves have $x=2.5,2.0,1.5,1.0,0.5$, and 0.0 . The steep dropoffs at $V \gtrsim 23.5 \mathrm{mag}$ (CFHT) and $V \gtrsim 24 \mathrm{mag}$ (Keck) are due to photometric incompleteness. Thus, to the completeness limits of our data, we find no evidence for a steeply rising luminosity function in Palomar 13 . On the contrary, the observed luminosity function appears quite flat, with $x \sim 0$, indicating a possible underabundance of low-mass stars.

tion and the preferential evaporation of low-mass stars. In short, the available data provide no reason to believe that the mass function of Palomar 13 is abnormal in any way, but firm conclusions must await deeper observations.

\subsection{Nonbaryonic Dark Matter?}

In $\S 5.2$, it was found that for an assumed mass-to-light ratio of $\Upsilon_{V}=2$ (i.e., a value appropriate for typical globular clusters), the tidal radius of Palomar 13 predicted by equation (9) was in good agreement with the tidal radius of the low-concentration $(c \sim 0.7) \quad$ King-Michie model adopted by Siegel et al. (2001). As discussed in $\S \S 3.5$ and 4, it is possible to obtain an excellent match to the observed surface brightness and surface density profiles using a KingMichie model that has $c=2.0 \pm 0.15$. In this case, the tidal radius of Palomar 13 is $r_{t} \sim 180 \mathrm{pc}$ (with a formal uncertainty of $\pm 23 \%$ based on our Monte Carlo simulations). From equation (9) we see that a tidal radius of this size suggests a mass of $M \sim 2 \times 10^{6} M_{\odot}$, i.e., considerably larger than our best estimate for the dynamical mass of Palomar 13 (assuming virial equilibrium) yet still far in excess of the expected mass. The actual uncertainties in the fitted tidal radii are difficult to gauge but are unlikely to be smaller than $\sim 50 \%$, which translates directly into a nearly a factor of 2 in mass. Thus, we conclude that the tidal radii inferred from the surface brightness and surface density profiles of Palomar 13 are (1) highly dependent on how one chooses to model the profile and (2) equally consistent with the tidal 
disruption of an otherwise normal globular cluster, or the presence of substantial dark matter component in this object.

In light of these results, it is natural to ask if the high velocity dispersion of Palomar 13 might also be the signature of a nonbaryonic dark matter halo, similar to those that are thought to belong to Local Group dSph galaxies (Aaronson 1983; Mateo 1998; cf. Klessen \& Kroupa 1998). This possibility is particularly topical given recent claims from CDM simulations that the halos of large galaxies may contain large numbers of low-mass "dark satellites" (e.g., Hirashita et al. 1998; Klypin et al. 1999; Bullock et al. 2000).

To examine this possibility, we consider a simple twocomponent model. First, we take the mass density profile of the luminous stellar component to be given by a Dehnen (1993) model,

$$
\rho_{*, m}(r)=[(3-\gamma) / 4]\left(\Upsilon \rho_{*, l}^{0} / \pi a^{3}\right)(r / a)^{-\gamma}(1+r / a)^{\gamma-4},
$$

where $\rho_{*, l}^{0}$ is the central luminosity density of this component, $a$ is its scale radius, and $\Upsilon$ is its mass-to-light ratio. By contrast, the dark halo is assumed to have the form proposed by Navarro, Frenk, \& White (1997):

$$
\rho_{d}(r)=\rho_{d}^{0}\left(r / r_{s}\right)^{-1}\left(1+r / r_{s}\right)^{-2},
$$

where $r_{s}$ is the scale radius of the dark halo, and $\rho_{d}^{0}$ is the central dark matter density. Once these density distributions are specified, the velocity dispersion profile follows from the Jeans equation

$$
\frac{d}{d r}\left[\rho_{*}(r) \sigma_{r}^{2}(r)\right]+\frac{2 \beta \rho_{*}(r) \sigma_{r}^{2}(r)}{r}=-\frac{G M(r)}{r^{2}} \rho_{*}(r),
$$

where $\sigma_{r}(r)$ is the radial component of the velocity dispersion and $M(r)$ is the combined masses of the luminous and dark components. For our purposes, we assume that the velocity dispersion tensor is isotropic (i.e., $\beta \equiv 0$ ). This theoretical velocity dispersion profile is then projected onto the plane of the sky and compared directly to the observed velocity dispersion.

Before doing so, we require estimates of $\gamma$ and $a$ from the surface brightness profile. The long-dashed curve in Figure 7 shows the best-fit Dehnen model, with $\gamma=1.46 \pm 0.06$ and $a=135^{\prime \prime} \pm 9^{\prime \prime}(15.9 \pm 1.0 \mathrm{pc})$, overlaid on the observed surface brightness profile. For an assumed mass-to-light ratio of $\Upsilon_{V}=2$, the total mass associated with this stellar component is $M=7 \times 10^{3} M_{\odot}$. We also assume that, in projection, the dark matter matches that of the surface brightness profile, giving $r_{s}=24^{\prime \prime} \pm 1^{\prime \prime}(2.8 \pm 0.1 \mathrm{pc})$. This Navarro-Frenk-White (NFW) model-fitted to the observed surface brightness profile - is shown by the solid curve in Figure 7. The central mass density of this model is then left as a free parameter that is adjusted until a reasonable match to the observed velocity dispersion is achieved. The corresponding velocity dispersion profile for a central mass density of $\rho_{d}^{0}=80 \pm 31 M_{\odot} \mathrm{pc}^{-3}$ is shown as the solid curve in Figure 8 (curve 3 ). This curve provides an adequate (although by no means unique) description of the observed velocity dispersion profile including the possible maximum at $R \sim 1^{\prime}$. Although the mass of an NFW profile diverges as $r \rightarrow \infty$, we note that over the range of our radial velocity and surface brightness observations, the implied mass-tolight ratio of this two component model is $\Upsilon_{V}=19_{-7}^{+8}$, about half that found using a King-Michie model but still well above that expected for a normal stellar population.

How does the central density of this putative dark halo compare to those found for the dSph satellites of the Milky Way? Table 1 of Mateo (1998) lists structural parameters for nine Galactic dSph galaxies having measured velocity dispersions: the central densities of these galaxies span the range $0.03 M_{\odot} \mathrm{pc}^{-3} \leq \rho^{0} \leq 0.6 M_{\odot} \mathrm{pc}^{-3}$, with a mean value of $\left\langle\rho^{0}\right\rangle=0.26 \pm 0.07 M_{\odot} \mathrm{pc}^{-3}$. Strictly speaking, these values refer to the combined densities of the dark and luminous components $\rho^{0}=\rho_{d}^{0}+\rho_{*, m}^{0}$. However, in view of the low stellar densities of these galaxies (e.g., $\left.\rho_{*, m}^{0} \sim 0.01 M_{\odot} \mathrm{pc}^{-3}\right), \rho^{0} \simeq \rho_{d}^{0}$ to good approximation. Thus, the dark matter density needed to explain the measured velocity dispersion of Palomar 13 exceeds that of typical dSph galaxies by more than 2 orders of magnitude.

Following Kormendy (1990), we show in Figures 12 and 13 the scaling relations between central density, velocity dispersion, $\sigma$, absolute blue magnitude, $M_{B}$, core radius, $r_{c}$, and central $B$-band surface brightness, $\mu_{B}$, for a sample of Local Group dSph galaxies (Mateo 1998), dwarf irregular and spiral galaxies (Côté, Carignan, \& Freeman 2000 and references therein), and Galactic globular clusters (Pryor \& Meylan 1993; Harris 1996). Note that the densities plotted here refer to those of the dark halos, with the exception of the globular clusters; for these objects, the densities are those calculated directly from the measured velocity dispersions. For Palomar 13, which is indicated by the large filled circle, we take the structural parameters for its putative dark matter halo to be those given by the best-fit NFW model. As these figures demonstrate, the central dark matter density in Palomar 13 is indeed much larger than those found for dwarf galaxies. On the other hand, it is also consistent with that expected from an extrapolation of galactic scaling relations to low luminosities. Indeed, it is remarkable that Palomar 13 falls so near the extrapolations of the tight scaling relations for dwarf galaxies, although this agreement cannot be taken as unambiguous evidence for a common origin since Palomar 13 also falls near the edges of the more heterogeneous distributions for Galactic globular clusters (and, in the scaling relations involving $\mu_{B}$, its location may be more consistent with the globular cluster distributions). Thus, the evidence from the observed scaling relations concerning the proper classification of Palomar 13 as a bona fide globular cluster or an extreme dark matter-dominated dwarf galaxy is ambiguous. In any event, we note that a naive extrapolation of the dwarf galaxy scaling relations to globular cluster scales predicts central densities for dark matter halos in these objects that are sufficiently low (i.e., $1 M_{\odot} \mathrm{pc}^{-3} \lesssim \rho_{d}^{0} \lesssim 100 M_{\odot} \mathrm{pc}^{-3}$ ) to make their detection extremely challenging in all but the faintest clusters. In other words, for the vast majority of globular clusters, the inferred dark matter densities would fall well below the baryonic densities.

Obviously, it would be of considerable interest to measure velocity dispersions for additional faint, low surface brightness clusters. An especially interesting target is AM 4, a cluster that shares many of the characteristics of Palomar 13, e.g., low-luminosity $\left(L_{V} \sim 400 L_{V, \odot}\right)$, low surface brightness $\left(\mu_{V, 0} \sim 24.75 \mathrm{mag} \operatorname{arcsec}^{-2}\right)$, and low metallicity $([\mathrm{Fe} / \mathrm{H}] \sim-2.0 \mathrm{dex})$. Like Palomar 13, it also occupies a relatively isolated location in the Galactic halo, with $R_{G}=25.5 \mathrm{kpc}$. The central velocity dispersion predicted by equation (7) for a "normal" globular cluster of this surface 

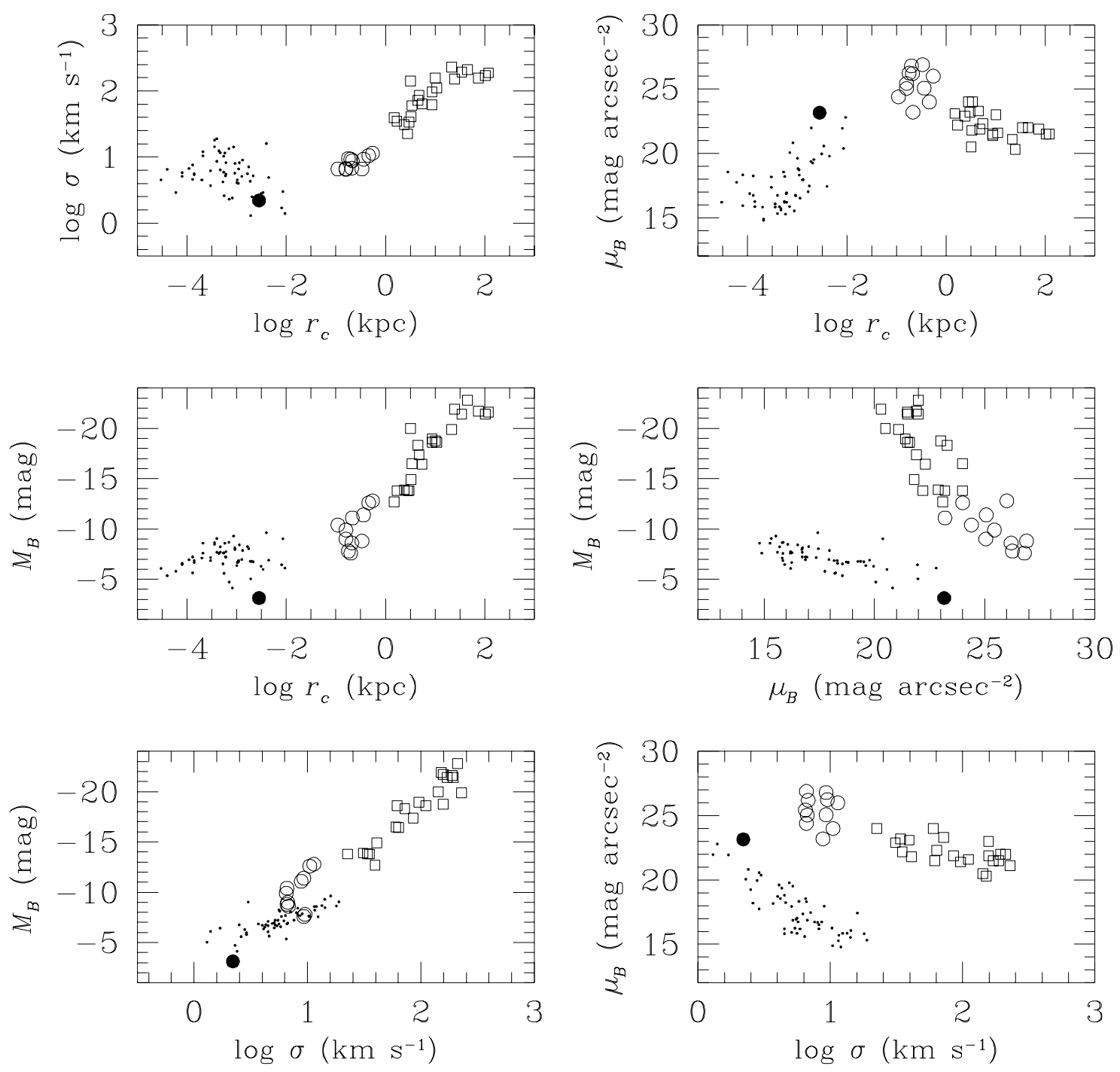

FIG. 12.-Scaling relations for stellar systems. Local Group dwarf spheroidal galaxies (data from Mateo 1998) are indicated by open circles. Open squares show the results for a sample of dwarf irregulars and spirals taken from Côté et al. (2000). Galactic globular clusters having measured velocity dispersions are shown by the dots (Pryor \& Meylan 1993; Harris 1996). Palomar 13 is indicated by the large filled circle.

brightness would be $\sigma_{p, 0} \lesssim 0.3 \mathrm{~km} \mathrm{~s}^{-1}$, and it would be interesting to see if this expectation is borne out by radial velocity measurements.

Three final scaling relations for dwarf galaxies are considered in Figure 14. In the upper panel of this figure, metallicity is plotted against absolute magnitude for $\mathrm{dE} / \mathrm{dSph}$ galaxies, along with the best-fit linear relation from Côté et al. (2000). Note that the relationship between metallicity and luminosity constitutes a key difference between dwarf galaxies and globular clusters; i.e., galaxies spanning a range of $\sim 10^{5}$ in luminosity follow a tight relation between metallicity and luminosity (e.g., Brodie \& Huchra 1991; Caldwell et al. 1992), whereas globular clusters obey no such correlation. While it is by no means clear that a simple extrapolation of this linear relation to $M_{V} \sim-4$ is justifiable, Palomar 13 is, nevertheless, considerably more metal-rich than might naively be expected for a dwarf of this luminosity. Furthermore, its CMD shows no evidence for an abundance spread, unlike Local Group dSph galaxies that often show inter- nal dispersions in metallicity of $\sim 0.35$ dex (e.g., Côté et al. 2000; Shetrone, Côté, \& Sargent 2001).

In the middle panel of Figure 14, the mass-to-light ratio is plotted against absolute magnitude for all Local Group $\mathrm{dE} / \mathrm{dSph}$ galaxies having measured velocity dispersions. The dashed curve shows the relation expected for dwarfs consisting of luminous stellar components with $\Upsilon_{V}=2$ that are embedded in dark matter halos of mass $M=2 \times 10^{7} M_{\odot}$, as proposed by Mateo et al. (1993). The mass-to-light ratio predicted for Palomar 13 by this relation would be $\Upsilon_{V} \sim 7000$, making it an extreme outlier. On the other hand, Palomar 13 does seem to follow the correlation between mass-to-light ratio, metallicity, and central surface brightness for Local Group dwarfs, shown in the lower panel of Figure 14 (Prada \& Burkert 2002). We can only conclude that the evidence regarding a possible connection between Palomar 13 and dark matter-dominated dwarf galaxies is ambiguous and that, in light of the complexity of gas cooling, star formation and feedback in low-mass systems 

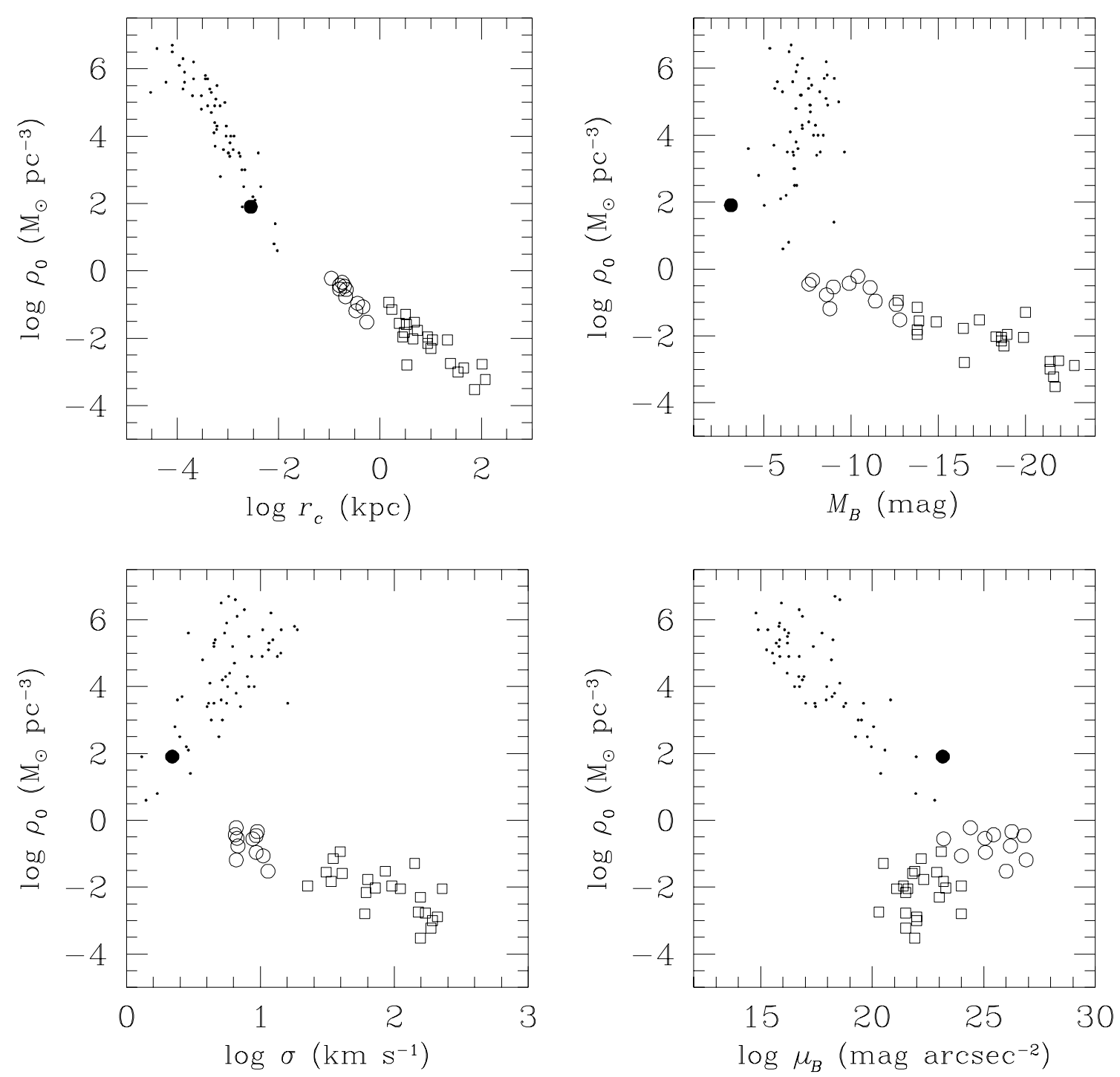

FIG. 13.-Same as Fig. 12, but for additional scaling relations for galaxies and Galactic globular clusters

(see, e.g., Mateo 1998, Fig. 8), a definitive conclusion is not possible at the present time.

\subsection{Modified Newtonian Dynamics?}

A much different interpretation of the large velocity dispersion of $\mathrm{dSph}$ galaxies has been proposed by Milgrom (1983a), who suggested that at low accelerations, $a \ll a_{0}$, Newtonian gravity should be revised to include a repulsive term. If this modified theory of Newtonian dynamics (MOND) is correct, then there would be no need to invoke dark matter as an explanation for the high-velocity dispersions of dwarf galaxies (Milgrom 1983b, 1995). But if MOND is to be a viable alternative to dark matter, then it must apply to all systems in which the acceleration falls below the characteristic MOND acceleration, $a_{0} \simeq 1.2 \times 10^{-8} \mathrm{~cm} \mathrm{~s}^{-2}$, a value chosen to match the rotation curves of low-surface brightness galaxies (Begeman, Broeils, \& Sanders 1991; McGaugh \& de Blok 1998). Since this condition is met for Palomar 13, we now examine its internal dynamics within the MOND framework.
There are two relevant accelerations for Palomar 13: the acceleration arising from the internal mass distribution and an external component due to the Milky Way. We approximate the internal acceleration as $a_{\text {int }}=G M(r) / r^{2}$, where $M(r)$ is mass enclosed with a radius, $r$, for the best-fit King model shown in Figure 7. For an assumed mass-to-light ratio of $\Upsilon_{V}=2$, the internal acceleration reaches a maximum value of $a_{\text {int }} \simeq 1 \times 10^{-9} \mathrm{~cm} \mathrm{~s}^{-1}$ at $r \simeq r_{c}$. The external acceleration may be approximated as $a_{\mathrm{ext}}=\Theta_{c}^{2} / R_{G}$, or $a_{\mathrm{ext}}=6 \times 10^{-9} \mathrm{~cm} \mathrm{~s}^{-1}$ for $\Theta_{c}=220 \mathrm{~km} \mathrm{~s}^{-1}$ and $R_{G}=25.3$ kpc. Thus, according to MOND, Palomar 13 is in the "quasi-Newtonian weak-field limit" since $a_{\text {int }}<a_{\text {ext }}<a_{0}$. In this regime, the Newtonian mass is related to the MOND mass by a factor, $\mu$, which falls in the range $1 \lesssim \mu \lesssim a_{0} / a_{\text {ext }}$ (Gerhard \& Spergel 1992). From Table 3, the best-fit KingMichie model has $\Upsilon_{V} \simeq 40_{-17}^{+24}$ in solar units. Thus, the mass-to-light ratio in MOND would be reduced by - at most $-\mathrm{a}$ factor of $a_{0} / a_{\mathrm{ext}}$, giving $\Upsilon_{V} \simeq 20_{-9}^{+12}$. Apparently, the high mass-to-light ratio of Palomar 13 persists (at the $2 \sigma$ level) even within the MOND framework. We conclude that this explanation appears less appealing than those involving tidal disruption or dark matter. 

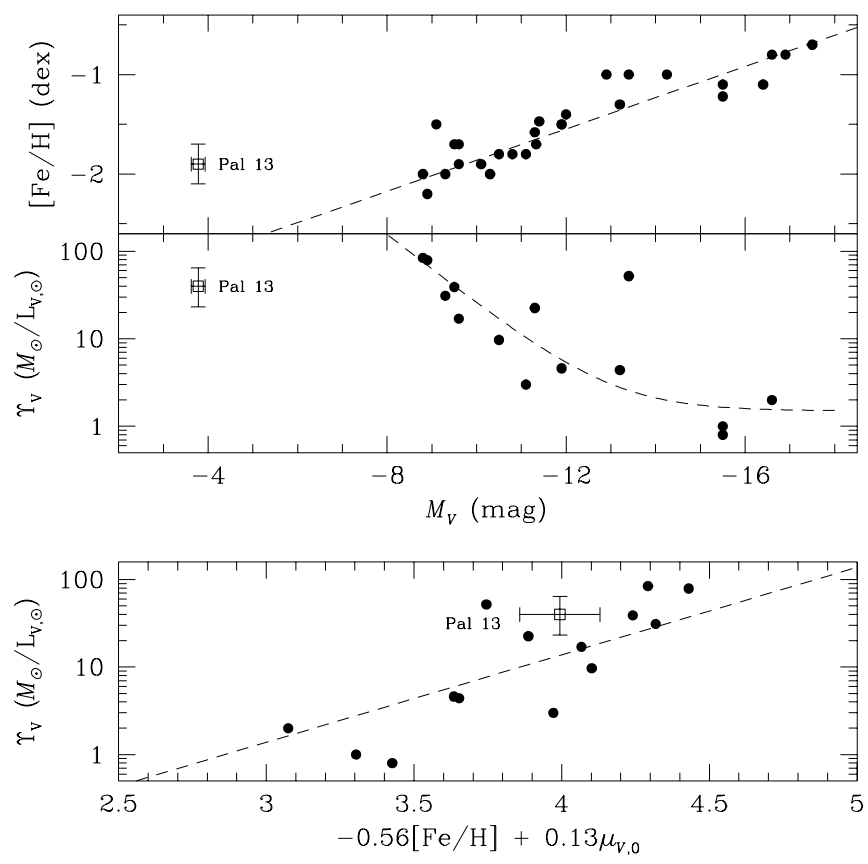

FIg. 14.-Top: Metallicity-luminosity relation for $\mathrm{dE} / \mathrm{dSph}$ galaxies ( filled circles). Palomar 13 is indicated by the open square. The dashed line shows the best-fit relation from Côté et al. (2000), extrapolated to faint magnitudes. Middle: $V$-band mass-to-light ratio, $\Upsilon_{V}$, vs. absolute visual magnitude for Local Group dE/dSph galaxies (see Côté et al. 1999). The dashed curve shows the relation for dwarf consisting of luminous stellar components with $\Upsilon_{V}=2$ that are embedded in dark matter halos of mass $M=2 \times 10^{7} M_{\odot}$. Palomar 13 obeys neither this, nor the above, empirical scaling relation for dwarf galaxies. Bottom: Correlation between mass-tolight ratio, metallicity, and central $V$-band surface brightness for Local Group $\mathrm{dE} / \mathrm{dSph}$ galaxies ( filled circles). The dashed line shows the linear relation proposed by Prada \& Burkert (2002). Palomar 13 is in reasonable agreement with the proposed correlation.

\section{SUMMARY AND CONCLUSIONS}

An imaging and spectroscopic survey of globular clusters in the outer halo of the Milky Way has shown Palomar 13 to exhibit several puzzling features.

From an abundance analysis of our HIRES spectra for a single RGB star and isochrone fitting to the CMD, we estimate the metallicity of Palomar 13 to be $[\mathrm{Fe} / \mathrm{H}]=$ $-1.9 \pm 0.2$ dex. We find a true distance modulus of $(m-M)_{0}=16.93 \pm 0.1 \mathrm{mag}$ and a distance of $R_{S}=$ $24.3_{-1.1}^{+1.2} \mathrm{kpc}$, placing Palomar 13 squarely in the outer halo. With $M_{V}=-3.8 \mathrm{mag}$, this is the lowest luminosity object in our radial velocity survey of halo clusters. The sparsely populated red giant and horizontal branches preclude a precise age determination, but a $14 \mathrm{Gyr}$ isochrone is found to provide a reasonable match to the observed fiducial sequences. In terms of its stellar populations, Palomar 13 therefore appears typical of globular clusters in the outer halo.
However, from a sample of 21 probable members with radial velocity measurements from HIRES, we find the systemic velocity and intrinsic velocity dispersion of Palomar 13 to be $\left\langle v_{r}\right\rangle_{s}=24.1 \pm 0.5 \mathrm{~km} \mathrm{~s}^{-1}$ and $\sigma_{p}=2.2 \pm 0.4 \mathrm{~km}$ $\mathrm{s}^{-1}$, respectively. This velocity dispersion is roughly 4 times higher than that expected on the basis of the observed luminosity and concentration. Taken at face value, this dispersion suggests a mass-to-light ratio of $\Upsilon_{V}=40_{-17}^{+24}$, with the exact value depending on the details of the modeling. This mass-to-light ratio is far higher than those found for globular clusters but is comparable to those of Galactic dSph galaxies. The surface density profile is also found to be anomalous among Galactic globular clusters, in that it shows a significant number of apparent member stars beyond the tidal radius of a low-concentration King-Michie model (i.e., $c \sim 0.7$, as has usually been claimed for Palomar 13). On the other hand, an excellent fit to the surface brightness profile over all radii is provided by a King-Michie model with $c \sim 2$, as well as by models of the type suggested by Dehnen (1993) and Navarro et al. (1997).

Based on the measured velocity dispersion and surface density profile, two possibilities present themselves: either Palomar 13 has recently undergone catastrophic heating and mass loss, as might be expected on the basis of its orbital properties, or its spatial extent, central concentration, and mass have been greatly underestimated in previous studies. Other factors, such as a large fraction of spectroscopic binary stars or the presence of a large number of heavy remnants, may contribute to Palomar 13's unusual properties but are, on their own, unlikely to provide a complete explanation of the observed structural and kinematic properties. Additional observations - particularly deep imaging with $H S T$ and ground-based mosaic cameras, and radial velocity measurements for an expanded sample of stars at large radii-should help discriminate between the various possibilities. In any event, it is clear that Palomar 13 merits further study, as it offers new insights into the structure and formation of the Galactic halo.

We thank Tad Pryor and an anonymous referee for numerous helpful comments. We also thank Gilles Bergond for his assistance with the CFHT observations, and Dean McLaughlin for providing some of the software used in the analysis. P. C. gratefully acknowledges support provided by the Sherman M. Fairchild Foundation during the initial stages of this work. S. J. G. acknowledges partial support from the Bressler Foundation. This work was based in part on observations obtained at the W. M. Keck Observatory, which is operated jointly by the California Institute of Technology and the University of California. We are grateful to the W. M. Keck Foundation for their vision and generosity.
Aaronson, M. 1983, ApJ, 266, L11

Anders, E., \& Grevesse, N. 1989, Geochim. Cosmochim. Acta, 53, 197

Bahcall, J. N. 1986, ARA\&A, 24, 577

Bahcall, J. N., \& Soneira, R. M. 1980, ApJS, 44, 73

Begeman, K. G., Broeils, A. H., \& Sanders, R. H. 1991, MNRAS, 249, 523

Bergbusch, P. A., \& VandenBerg, D. A. 1992, ApJS, 81, 163

Borissova, J., Markov, H., \& Spassova, N. 1997, A\&AS, 121, 499

Brodie, J. P., \& Huchra, J. P. 1991, ApJ, 379, 157

Bullock, J. S., Kravtsov, A. V., \& Weinberg, D. H. 2000, ApJ, 539, 517

Caldwell, N., Armandroff, T. E., Seitzer, P., \& Da Costa, G. S. 1992, AJ, 103,840

Canterna, R., \& Schommer, R. A. 1978, ApJ, 219, L119

\section{REFERENCES}

Cen, R. 2001, ApJ, 560, 592

Ciatti, F., Rosino, L., \& Sussi, M. 1965, K1. Veroff. Remeis-Sternwarte Bamberg, 4, 40, 228

Cohen, J. G. 1992, ApJ, 400, 528

Combes, F., Leon, S., \& Meylan, G. 1999, A\&A, 352, 149

Côté, P. 1999, AJ, 118, 406

Côté, P., Markze, R. O., West, M. J., \& Minniti, D. 2000, ApJ, 533, 869

Côté, P., Mateo, M., Olszewski, E. W., \& Cook, K. H. 1999, ApJ, 526, 147

Côté, P., Pryor, C., McClure, R. D., Fletcher, J. M., \& Hesser, J. E. 1996, AJ, 112,565

Côté, S., Carignan, C., \& Freeman, K. C. 2000, AJ, 120, 3027 
Cuillandre, J.-C., Luppino, G., Starr, B., \& Isani, S. 2000, Proc. SPIE, 4008, 1010

Davé, R., Spergel, D. N., Steinhardt, P. J., \& Wandelt, B. D. 2001, ApJ, 547,574

Dehnen, W. 1993, MNRAS, 265, 250

Dehnen, W., \& Binney, J. J. 1998, MNRAS, 298, 387

Dekel, A., \& Silk, J. 1986, ApJ, 303, 39

Dinescu, D. I., Majewski, S. R., Girard, T. M., \& Cudworth, K. M. 2001, AJ, 122, 1916

Djorgovski, S. G. 1991, in ASP Conf. Ser. 13, The Formation and Evolution of Star Clusters, ed. K. Janes (San Francisco: ASP), 112

Durrell, P. R., McLaughlin, D. E., Harris, W. E., \& Hanes, D. A. 1996, ApJ, 463, 543

Feltzing, S., Gilmore, G., \& Wyse, R. F. G. 1999, ApJ, 516, L17

Fich, M., \& Tremaine, S. 1991, ARA\&A, 29, 409

Friel, E., Kraft, R. P., Suntzeff, N. B., \& Carbon, D. F. 1982, PASP, 94, 873

Gunn, J. E., \& Griffin, R. F. 1979, AJ, 84, 752

Gerhard, O. E., \& Spergel, D. N. 1992, ApJ, 397, 38

Harris, W. E. 1996, AJ, 112, 1487

Heggie, D. C., \& Hut, P. 1996, in IAU Symp. 174, Dynamical Evolution of Star Clusters: Confrontation of Theory and Observations, ed. P. Hut \& J. Makino (Dordrecht: Kluwer), 303

Hills, J. G. 1984, AJ, 89, 1811

Hirashita, H., Takeuchi, T. T., \& Tamura, N. 1998, ApJ, 504, L83

Hut, P., et al. 1992, PASP, 104, 981

Idiart, Barbuy, B., Perrin, M. N., Ortolani, S., Bica, E., \& Renzini, A. 2002, A\&A, 381, 472

Johnston, K. V., Choi, P., \& Guhathakurta, P. 2001, AJ, submitted (astro$\mathrm{ph} / 0111466$ )

Johnston, K. V., Sigurdsson, S., \& Hernquist, L. 1999, MNRAS, 302, 771

Kells, W., Dressler, A., Sivaramakrishnan, A., Carr, D., Koch, E., Epps, H., Hilyard, D., \& Pardeilhan, G. 1998, PASP, 110, 1487

King, I. R. 1962, AJ, 67, 471

Kinman, T. D., \& Rosino, L. 1962, PASP, 74, 499

Klessen, R. S., \& Kroupa, P. 1998, ApJ, 498, 143

Klypin, A. A., Kravtsov, A. V., Valenzuela, O., \& Prada, F. 1999, ApJ, 522,82

Kormendy, J. 1990, in ASP Conf. Ser. 10, Evolution of the Universe of Galaxies, ed. R. G. Kron (San Francisco: ASP), 33

Krisciunas, K. 1997, PASP, 109, 1181

Kulkarni, S. R., \& Anderson, S. B. 1996, in IAU Symp. 174, Dynamical Evolution of Star Clusters: Confrontation of Theory and Observations, ed. P. Hut \& J. Makino (Dordrecht: Kluwer), 181

Kunkel, W. E. 1979, ApJ, 228, 718

Landolt, A. U. 1992, AJ, 104, 340

Leon, S., Meylan, G. \& Combes, F. 2000, A\&A, 359, 907

Lynden-Bell, D. 1976a, MNRAS, 174, 695 .1976b, R. Greenwich Obs. Bull., 182, 241

Lynden-Bell, D., \& Lynden-Bell, R. M. 1995, MNRAS, 275, 429

Majewski, S. R. 1994, ApJ, 431, L17

Majewski, S. R., et al. 2001, preprint (astro-ph/0109492)

Mateo, M. 1998, ARA\&A, 36, 435

Mateo, M., Harris, H. C., Nemec, J., \& Olszewski, E. W. 1990, AJ, 100, 469

Mateo, M., Olszewski, E., Pryor, C., Welch, D. L., \& Fischer, P. 1993, AJ, 105,510

Mateo, M., Olszewski, E., Welch, D. L., Fischer, P., \& Kunkel, W. 1991, AJ, 102,914

Mayor, M., et al. 1984, A\&A, 134, 118
McGaugh, S. S., \& de Blok, W. J. G. 1998, ApJ, 499, 66

McWilliam, A., Preston, G., Sneden, C., \& Searle, L. 1995, AJ, 109, 2757

Meylan, G. 1987, A\&A, 184, 144 1989, A\&A, 214, 106

Mihalas, D., \& Binney, J. 1981, Galactic Astronomy: Structure and Kinematics (2d ed.; San Francisco: Freeman)

Milgrom, M. 1983a, ApJ, 270, 365

1983b, ApJ, 270, 37

1995, ApJ, 455, 439

Moore, B. 1996, ApJ, 461, L13

Moore, B., Calcáneo-Roldán, C., Stadel, J., Quinn, T., Lake, G., Ghinga, S., \& Governato, F. 2001, Phys. Rev. D, 64, 063508

Moore, B., Ghinga, S., Governato, F., Lake, G., Quinn, T., Stadel, J., \& Tozzi, P. 1999, ApJ, 524, L19

Navarro, J. F. Frenk, C. S., \& White, S. D. M. 1997, ApJ, 490, 493

Oke, J. B., Cohen, J. G., Carr, M., Cromer, J., Dingizian, A., Harris, F. H., Labrecque, S., Lucinio, R., Schaal, W., Epps, H., \& Miller, J. 1995 , PASP, 107, 375

Olszewski, E. W., Pryor, C., \& Schommer, R. B. 1993, in ASP Conf. Ser. 48, The Globular Cluster-Galaxy Connection, ed. G. Smith \& J. Brodie (San Francisco: ASP), 99

Ortolani, S., Rosino, L., \& Sandage, A. 1985, AJ, 90, 473

Peebles, P. J. E. 1984, ApJ, 277, 470

Piatek, S., \& Pryor, C. 1995, AJ, 109, 107

Prada, F., \& Burkert, A. 2002, ApJ, 564, L73

Pryor, C., Latham, D. W., \& Hazen, M. L. 1988, AJ, 96, 123

Pryor, C., \& Meylan, G. 1993, in ASP Conf. Ser. 50, The Structure and Dynamics of Globular Clusters, ed. S. G. Djorgovski \& G. Meylan (San Francisco: ASP), 357

Racine, R. 1975, AJ, 80, 1031

Richer, H. B., et al. 1997, ApJ, 484, 741

Richstone, D. O., \& Tremaine, S. 1986, AJ, 92, 72

Romani, R. W., \& Weinberg, M. D. 1991, ApJ, 372, 487

Rosenblatt, E. I., Faber, S. M., \& Blumenthal, G. R. 1988, ApJ, 330, 191

Rubenstein, E. P., \& Bailyn, C. D. 1997, ApJ, 474, 701

Salpeter, E. E. 1955, ApJ, 121, 161

Schlegel, D. J., Finkbeiner, D. P., \& Davis, M. 1998, ApJ, 500, 525

Shetrone, M. D. Côté, P. \& Sargent, W. L. W. 2001, ApJ, 548, 592

Siegel, M. H., Majewski, S. R., Cudworth, K. M., \& Takamiya, M. 2001, AJ, 121,935

Silvestri, F., Ventura, P., D’Antona, F., \& Mazzitelli, I. 1998, ApJ, 509, 192

Sneden, C. 1973, Ph.D thesis, Univ. Texas, Austin

Stetson, P. B. 1993, in IAU Colloq. 136, Stellar Photometry: Current Techniques and Future Developments, ed. C. J. Butler \& I. Elliot (Cambridge: Cambridge Univ. Press), 291

Stetson, P. B., et al. 1999, AJ, 117, 247

Tonry, J., \& Davis, M. 1979, AJ, 84, 1511

Trager, S. C., King, I. R., \& Djorgovski, S. G. 1995, AJ, 109, 218

van den Bergh, S. 1994, AJ, 108, 2145

Verbunt, F. 2001, A\&A, 368, 137

Vesperini, E., \& Heggie, D. C. 1997, MNRAS, 289, 898

Vogt, S. S., Mateo, M., Olszewski, E. W., \& Keane, M. J. 1995, AJ, 109, 15

Vogt, S. S., et al. 1994, Proc. SPIE, 2198, 362

Webbink, R. F. 1985, in IAU Symp. 113, Dynamics of Star Clusters, ed. J. Goodman \& P. Hut (Dordrecht: Reidel), 541

West, M. J. 1993, MNRAS, 265, 755

Zinn, R., \& Diaz, A. I. 1982, AJ, 87, 1190 